\title{
Nonthermal radiation from jets of active galactic nuclei: Electrostatic bremsstrahlung as alternative to synchrotron radiation
}

\begin{abstract}
R. Schlickeiser ${ }^{\star}$
Institut für Theoretische Physik, Lehrstuhl IV: Weltraum- und Astrophysik, Ruhr-Universität Bochum, 44780 Bochum, Germany

Received 1 April 2003 / Accepted 4 July 2003

Abstract. The interaction of collimated relativistic hadronic and leptonic jet outflows from active galactic nuclei with the surrounding interstellar and intergalactic medium generates by two-stream instabilities huge intensities of subluminal electrostatic (Langmuir) wave fields in the co-moving outflow region with energy densities larger than the energy density in the magnetic field. We therefore revisit electrostatic bremsstrahlung, i.e. the inverse Compton scattering of electrostatic waves by energetic positrons and electrons to photons $\left(l+e \rightarrow t+e^{\prime}\right)$, as an alternative nonthermal radiation process to synchrotron radiation which in the literature sofar has been the standard low-frequency radiation process for radiation. It is known that this radiation process has similar polarisation properties as synchrotron radiation. By calculating Doppler boosted luminosity spectra in case of leptonic outflows we demonstrate that in jet outflow sources electrostatic bremsstrahlung by swept-up relativistic electrons is stronger than synchrotron radiation from the same electrons in the radio to optical frequency band, whereas the optically thick bulk electrostatic bremsstrahlung from the non-relativistic pair outflow plasma is typically a factor $10^{10}$ smaller. Our analysis is based on photon angle integrated monochromatic approximations of the electrostatic bremsstrahlung power and synchrotron radiation power in a plasma, which are constructed from moments of the respective radiation powers of single electrons and positrons. Specialising to the specific subluminal electrostatic wave distribution and the swept-up relaivistic electrons in the leptonic jet model of Schlickeiser et al. (2002) we calculate the expected Doppler boosted radiation intensities from bulk electrostatic bremsstrahlung, relativistic electron bremsstrahlung and synchrotron radiation for standard inner jet parameters. This requires to investigate in detail (i) the equilibrium distribution of the radiating electrons and (ii) the emission and absorption coefficients of each radiation process. An attractive feature of electrostatic bremsstrahlung mechanism is the fact that the interaction of the jet outflow with the surrounding ambient medium generates both the target electrostatic plasma waves and the radiating swept-up relativistic electrons. It does not rely on the existence of large intrinsic ordered magnetic fields to account for strong emission due to the synchrotron radiation process. This might be of importance also for nonthermal emission far away from the central jet engine.
\end{abstract}

Key words. galaxies: active - galaxies: jets - radiation mechanisms: non-thermal - plasmas

\section{Introduction}

It is generally accepted that relativistic jet outflows power the nonthermal emission from active galactic nuclei (AGN), irrespective of the composition of these jets - leptonic (i.e. positron and electron pairs) or hadronic (protons and electrons). Recently in a series of papers (Pohl \& Schlickeiser 2000; Pohl et al. 2002; Schlickeiser et al. 2002) we investigated the microphysical details of the conversion process of the kinetic energy in collimated relativistic hadronic and leptonic jet outflows into radiation through interactions with the ambient interstellar medium. Viewed from the coordinate system comoving with the outflow, the interstellar protons and electrons represent a proton-electron beam propagating with relativistic speed in the jet plasma. We demonstrated that this beam

\footnotetext{
* e-mail: rsch@tp4.ruhr-uni-bochum.de
}

excites both electrostatic and low-frequency magnetohydrodynamic Alfven-type waves via a two-stream instability in the jet background plasma. For standard AGN jet outflow and environment parameters the initial beam distributions of interstellar protons and electrons quickly relax to plateau-distributions in parallel momentum, transferring thereby one-half of the initial energy density of the beam particles to electric field fluctuations of the generated electrostatic turbulence ${ }^{1}$. On considerably longer time scales, the plateaued interstellar electrons and protons isotropise by their self-generated transverse turbulence, and are thus picked-up in the outflow jet plasma.

${ }^{1}$ In our study we will frequently refer to the work of Schlickeiser et al. (2002 - hereafter referred to as Paper S), using the same notation and referring to specific results of that paper e.g. Eq. (S-18) refers to Eq. (18) of Paper S. 
It is the purpose of this study to investigate the further fate of the generated electrostatic waves whose energy density (Eq. (S-69) combined with the density transformation formula $n_{\mathrm{i}}=\Gamma n_{\mathrm{i}}^{*}$ of observer's system and comoving densities, respectively)

$$
\begin{aligned}
U_{\mathrm{L}} & =\frac{1}{2} \Gamma n_{\mathrm{i}} m_{\mathrm{p}} c^{2}=\frac{1}{2} \Gamma^{2} n_{\mathrm{i}}^{*} m_{\mathrm{p}} c^{2} \\
& =7.3 \Gamma_{2}^{2}\left(n_{\mathrm{i}}^{*} / 1 \mathrm{~cm}^{-3}\right) \mathrm{erg} \mathrm{cm}^{-3}
\end{aligned}
$$

equals half of the initial energy density of the beam particles. For relativistic $\left(\Gamma=10^{2} \Gamma_{2}\right)$ outflows $U_{\mathrm{L}}$ can be significantly larger than the magnetic energy density $U_{\mathrm{B}}=B_{0}^{2} /(8 \pi)=$ $0.04\left(B_{0} / 1 \text { Gauss }\right)^{2} \mathrm{erg} \mathrm{cm}^{-3}$ of the jet plasma (for a justification of the chosen physical parameters see Sect. 1.2 below). Under these conditions, the generation of nonthermal photon radiation by electrostatic bremsstrahlung (Gailitis \& Tsytovich 1964; Colgate 1967); Melrose (1971, 1971), which is the inverse Compton scattering off electrostatic plasma waves into electromagnetic waves by energetic electrons and positrons, is more important by a factor $U_{\mathrm{L}} / U_{\mathrm{B}}$ than synchrotron radiation. All existing models in the literature (Maraschi et al. 1992; Dermer \& Schlickeiser 1993; Mannheim 1993; Sikora et al. 1994; Bloom \& Marscher 1996; Böttcher et al. 1997; Dermer et al. 1997; Tavecchio et al. 1998) (see Böttcher 2002 and Sikora \& Madejski 2002 for recent reviews), for the broadband nonthermal photon emission from jets of active galactic nuclei assume synchrotron radiation to be the most important nonthermal radiation mechanism at radio to X-ray frequencies. Although the polarisation properties of electrostatic bremstrahlung are similar to that of synchrotron radiation (Chiuderi \& Veltri 1974; Windsor \& Kellogg 1974), this radiation process has not received much attention in the literature. This was probably prompted by Melrose's (1971, p. 135) remark that it would require exceptional circumstances for the energy density in Langmuir (electrostatic) waves to exceed the energy density in the magnetic field. However, our estimate in Eq. (1) shows that in the two-stream dissipation process of relativistic outflows indeed huge Langmuir wave energy densities occur.

\subsection{Motivation and scope of this study}

The following study is motivated by the result of Paper $\mathrm{S}$ that in the interaction of the ambient fully-ionised plasma with the leptonic jet outflow (viewed from the comoving outflow system the ambient plasma penetrates the leptonic jet plasma) the twostream instabilities engender extremely powerful Langmuir waves which in turn produces huge amounts of electrostatic bremsstrahlung. Throughout this work this scenario is assumed to be given, and we investigate its consequences especially for the resulting spectral appearance of these sources.

We are aware that the treatment in Paper S of the interaction of the leptonic jet outflow with the ambient plasma in some dynamical aspects is not complete. In particular, the study in Paper S neglects the potential formation of a bow shock from reflected paricles (see Vainio et al. 2003) propagating ahead of the jet which should displace the ambient medium and might diminish the ambient medium density in the immediate vicinity of the jet itself ${ }^{2}$. As a consequence then, the density of interstellar particles $n_{\mathrm{i}}$ penetrating into the outflow plasma will be reduced, yielding according to Eq. (1) a reduced level of Langmuir waves and a reduced level of swept-up interstellar particles in the jet plasma. If one draws analogies to the interaction of nonrelativistic outflows, probably a shear zone at a contact discontinuity will develop through which the shocked ambient plasma might mix with the shocked jet plasma and where two-stream instabilities could be important - but this would probably involve only a small fraction of the jet.

If the density $n_{\mathrm{i}}$ of penetrating particles is smaller than the value

$n_{\mathrm{i}, \mathrm{B}}=5.5 \times 10^{-3}\left(B_{0} / 1 \text { Gauss }\right)^{2} \Gamma_{2}^{-1} \mathrm{~cm}^{-3}$

the energy density $U_{\mathrm{L}}$ in Langmuir waves becomes smaller than the magnetic field energy density $U_{\mathrm{B}}$. The optically thin intensities of relativistic electrostatic bremsstrahlung $I_{\mathrm{eb}}$ (Sect. 8), optically thin synchrotron radiation $I_{\mathrm{sy}}$ (Sect. 8) and SSC-radiation $I_{\mathrm{SSC}}$ in a homogenous one-zone emission region, which is a thick target for the relativistic electrons, scale as

$$
\begin{aligned}
& I_{\mathrm{eb}} \propto n_{\mathrm{i}} \frac{U_{\mathrm{L}}}{U_{\mathrm{L}}+U_{\mathrm{B}}}=n_{\mathrm{i}} \frac{n_{\mathrm{i}}}{n_{\mathrm{i}}+n_{\mathrm{i}, \mathrm{B}}} \simeq \begin{cases}\frac{n_{\mathrm{i}}^{2}}{n_{\mathrm{i}, \mathrm{B}}} & \text { for } n_{\mathrm{i}} \leq n_{\mathrm{i}, \mathrm{B}} \\
n_{\mathrm{i}} & \text { for } n_{\mathrm{i}} \geq n_{\mathrm{i}, \mathrm{B}}\end{cases} \\
& I_{\mathrm{sy}} \propto n_{\mathrm{i}} \frac{U_{\mathrm{B}}}{U_{\mathrm{L}}+U_{\mathrm{B}}}=\frac{n_{\mathrm{i}} n_{\mathrm{i}, \mathrm{B}}}{n_{\mathrm{i}}+n_{\mathrm{i}, \mathrm{B}}} \simeq \begin{cases}n_{\mathrm{i}} & \text { for } n_{\mathrm{i}} \leq n_{\mathrm{i}, \mathrm{B}} \\
n_{\mathrm{i}, \mathrm{B}} & \text { for } n_{\mathrm{i}} \geq n_{\mathrm{i}, \mathrm{B}}\end{cases}
\end{aligned}
$$

and

$$
\begin{aligned}
I_{\mathrm{SSC}} & \propto \frac{n_{\mathrm{i}}}{U_{\mathrm{L}}+U_{\mathrm{B}}} I_{\mathrm{sy}}=n_{\mathrm{i}}^{2} \frac{U_{\mathrm{B}}}{\left(U_{\mathrm{L}}+U_{\mathrm{B}}\right)^{2}} \\
& =\frac{n_{\mathrm{i}}^{2} n_{\mathrm{i}, \mathrm{B}}^{2}}{U_{\mathrm{B}}\left(n_{\mathrm{i}}^{2}+n_{\mathrm{i}, \mathrm{B}}^{2}\right)} \begin{cases}\frac{n_{\mathrm{i}}^{2}}{U_{\mathrm{B}}} & \text { for } n_{\mathrm{i}} \leq n_{\mathrm{i}, \mathrm{B}} \\
\frac{n_{\mathrm{i}, \mathrm{B}}}{U_{\mathrm{B}}} & \text { for } n_{\mathrm{i}} \geq n_{\mathrm{i}, \mathrm{B}}\end{cases}
\end{aligned}
$$

As can be seen, the relative intensities of these three radiation processes depend crucially on the density of penetrating particles in the jet. This emphasizes again the importance of investigating the dynamical processes associated withe the interaction of relativistic outflow with the surrounding medium. Some aspects are under investigation, see the study of Vainio et al. (2003); but as noted above, they are not the subject of the present study. A major difficulty is our current poor understanding of the physics of relativistic shocks in a almost collisionfree plasma environment. The formation of shock waves and contact discontinuities is theoretically and observationally (by in situ observations in the heliosphere) fairly well developed for non-relativistic shock speeds and collision-dominated plasma environment, allowing to use the ideal MHD shock equations; but in the case of relativistic shock speeds in a collision-poor plasma environment, where a full kinetic description is necessary, this is not the case. We hope that our study here will prompt such investigations in the future.

Another argument in favour of penetrating ambient plasma particles arises from the solar system analogy of interstellar pick-up ions entering the heliosphere (Fisk et al. 1974; Möbius et al. 1985). Most likely, also the ambient interstellar medium

\footnotetext{
${ }^{2}$ I am grateful to the referee for also emphasizing this point.
} 
in AGNs is a partially ionised plasma containing a large fraction of neutral atoms. As in the case of heliospheric pick-up ions, these neutrals easily enter the jet outflow plasma, and are ionised by both, charge-exchange as well as collissional and radiative interaction processes (Rucinski et al. 1996) with the outflow plasma and the radiation field of the jet plasma, respectively. After ionisation they form an anisotropic backward propagating beam distribution of the type considered in Paper S.

\subsection{Range of application}

Under the assumption, that indeed the huge Langmuir wave energy densities of Eq. (1) are generated in the interaction of the jet outflow with the ambient medium, we will demonstrate in the following that electrostatic bremsstrahlung is more important than synchrotron radiation. Consequently, the nonthermal radiation from jets of active galactic nuclei then have to be interpreted in a different way then before.

Here we are primarily interested in the correlation of the high-energy gamma-ray emission with the low-frequency emission in the infrared-optical-UV band. In current models of jet radiation (see references above), the low-frequency emission is interpreted as Doppler-boosted synchrotron radiation of the jet electrons and positrons, while the high-energy radiation is often interpreted as Doppler-boosted inverse Compton scattering of low-frequency photons by the same jet electrons and positrons, including the synchrotron photons (SSC process) or external photons coming directly from the acrretion disk or being reprocessed accretion disk photons. Therefore we will chose plasma parameter values (density of jet electrons, jet radius and size, magnetic field strength, outflow Lorentz factors) that are necessary to reproduce the high-energy gamma radiation from these sources. These plasma parameters are constrained by the highest frequencies observed, by the observed time variability of these sources down to the sub-hr time scale, gamma-ray transparancy arguments and limits on the inverse Compton optical depth of the relativistic electrons and positrons (see e.g. Schlickeiser 1996). These constraints lead to standard jet outflow parameters like e.g. the jet radius of $10^{14} \mathrm{~cm}$ and jet Lorentz factors of $100 \Gamma_{2}$, which are very small and very large, respectively, compared to values backed by VLBI-radio observations of AGNs. However, such parameter values apply to jet distances close to the central engine; in fact, they are necessary to meet the time variability constraints of $\mathrm{TeV}$ gamma-ray emission and to generate $\mathrm{TeV}$ gamma-ray photons both from the inverse Compton process and from $\pi^{0}$ decay within the relativistic hadronic pick-up model (Pohl \& Schlickeiser 2000). In order to apply our results also to other jet distances with different plasma parameters we will, whenever possible, note in our formulas the scaling with these parameter values.

\subsection{Organization of the paper}

In order to demonstrate the importance of electrostatic bremsstrahlung over synchrotron radiation we have to derive formulas for the spontaneous emission coefficient and the absorption coefficient of electrostatic bremsstrahlung for the case of an anisotropic subluminal distribution of target Langmuir photons. For anisotropic, subluminal distributions these are not available in the literature; we therefore start in three rather technical sections (Sects. 2-4) with the derivation of the radiation power of electrostatic bremsstrahlung (Sect. 2) of a single electron with velocity $\boldsymbol{\beta}$ scattering off electrostatic plasma waves with the distribution $N_{\mathrm{L}}\left(\boldsymbol{k}_{\mathrm{L}}\right)$, where $\boldsymbol{k}_{\mathrm{L}}$ denotes the wave vector of the electrostatic waves, to generate photons of frequencies $\omega$ moving into the directions $(\theta, \phi)$. From the associated electron energy loss rate and the mean energy of the generated photons (Sect. 3) we construct the monochromatic approximation of the radiation power for sublubminal Langmuir waves (Eq. (57)) which is very useful to model the electrostatic bremsstrahlung spectra from interacting particles with a broad energy distribution function (Sect. 4).

Readers interested only in the astronomical applications may skip these sections and directly proceed to Sect. 5 where we calculate the electrostatic bremsstrahlung from the isotropic bulk pair jet plasma. After determining in Sect. 6 the equilibrium distribution function of the isotropised swept-up relativistic electrons, we calculate in Sect. 7 their electrostatic bremsstrahlung and compare with their synchrotron radiation. In Sect. 8 we determine the Doppler boosted intensities from the three (bulk and relativistic electrostatic bremsstrahlung, synchrotron radiation) radiation processes. Our results are summarised in Sect. 9.

\section{Electrostatic bremsstrahlung}

\subsection{Total and differential radiation power}

We consider a single electron or positron of velocity $\boldsymbol{\beta} c$ scattering off electrostatic waves with a distribution $N_{\mathrm{L}}\left(\boldsymbol{k}_{\mathrm{L}}\right)$ with respect to the wavenumber vector $\boldsymbol{k}_{\mathrm{L}}=k_{\mathrm{L}} \boldsymbol{\kappa}_{\mathrm{L}}$, yielding photons with wavenumber vector $\boldsymbol{k}=\boldsymbol{k} \boldsymbol{\kappa}$.

Acoording to Eqs. (4) and (5) of Melrose (1971) the total photon power radiated spontaneously is

$P(\boldsymbol{\beta})=\int \frac{\mathrm{d}^{3} \boldsymbol{k}}{(2 \pi)^{3}} \int \frac{\mathrm{d}^{3} \boldsymbol{k}_{\mathrm{L}}}{(2 \pi)^{3}} \hbar \omega w\left(\boldsymbol{\beta}, \boldsymbol{k}, \boldsymbol{k}_{\mathrm{L}}\right) N_{\mathrm{L}}\left(\boldsymbol{k}_{\mathrm{L}}\right)$

where the photon frequency is $\omega=2 \pi v=k c$ and where the probability of spontaneous emission, summed over the polarisations of the scattered electromagnetic waves, is given by

$$
\begin{aligned}
w\left(\boldsymbol{\beta}, \boldsymbol{k}, \boldsymbol{k}_{\mathrm{L}}\right)= & \frac{8 \sigma_{\mathrm{T}} c^{4}}{3 \pi} \frac{(2 \pi)^{3} \omega_{\mathrm{p}}}{\omega^{3}} \frac{1-\beta^{2}}{[1-\boldsymbol{\beta} \cdot \boldsymbol{\kappa}]^{4}} \\
& \times\left[[1-\boldsymbol{\beta} \cdot \boldsymbol{\kappa}]^{2}\left[1-\left(\boldsymbol{\beta} \cdot \boldsymbol{\kappa}_{\mathrm{L}}\right)^{2}\right]-\left(1-\beta^{2}\right)\right. \\
& \left.\times\left[\boldsymbol{\kappa} \cdot \boldsymbol{\kappa}_{\mathrm{L}}-\boldsymbol{\beta} \cdot \boldsymbol{\kappa}_{\mathrm{L}}\right]^{2}\right] \\
& \times \delta\left(\omega[1-\boldsymbol{\beta} \cdot \boldsymbol{\kappa}]-\omega_{\mathrm{p}}\left[1-\frac{k_{\mathrm{L}} c}{\omega_{\mathrm{p}}} \boldsymbol{\beta} \cdot \boldsymbol{\kappa}_{\mathrm{L}}\right]\right)
\end{aligned}
$$

where $\omega_{\mathrm{p}}$ denotes the plasma frequency of the background jet plasma, which equals the electron plasma frequency $\omega_{\mathrm{p}, \mathrm{e}}=$ $\sqrt{4 \pi e^{2} n_{\mathrm{b}} / m_{\mathrm{e}}}=5.64 \times 10^{8}\left(n_{\mathrm{b}, 10}^{*}\right)^{1 / 2} \Gamma_{2}^{-1 / 2} \mathrm{~Hz}$ in case of hadronic jets and $\sqrt{2} \omega_{\mathrm{p}, \mathrm{e}}$ in case of leptonic jets. $\sigma_{\mathrm{T}}$ is the Thomson 
cross section; the pair density, as measured in the observer's frame, is scaled as $n_{\mathrm{b}}^{*}=10^{10} n_{\mathrm{b}, 10}^{*} \mathrm{~cm}^{-3}$.

The energy density $U_{\mathrm{L}}$ in electrostatic waves is related to their spectral distribution $N\left(\boldsymbol{k}_{\mathrm{L}}\right)$ by

$$
\begin{aligned}
U_{\mathrm{L}}= & \int \frac{\mathrm{d}^{3} \boldsymbol{k}_{\mathrm{L}}}{(2 \pi)^{3}} \hbar \omega_{\mathrm{p}} N_{\mathrm{L}}\left(\boldsymbol{k}_{\mathrm{L}}\right) \\
= & \frac{\hbar \omega_{\mathrm{p}}}{(2 \pi)^{3}} \int_{0}^{2 \pi} \mathrm{d} \phi_{\mathrm{L}} \int_{-1}^{1} \mathrm{~d}\left(\cos \theta_{\mathrm{L}}\right) \\
& \times \int_{0}^{\infty} \mathrm{d} k_{\mathrm{L}} k_{\mathrm{L}}^{2} N_{\mathrm{L}}\left(k_{\mathrm{L}}, \theta_{\mathrm{L}}, \phi_{\mathrm{L}}\right)
\end{aligned}
$$

Using

$$
\int \frac{\mathrm{d}^{3} \boldsymbol{k}}{(2 \pi)^{3}}=\frac{1}{(2 \pi)^{3} c^{3}} \int_{0}^{\infty} \mathrm{d} \omega \omega^{2} \int_{0}^{2 \pi} \mathrm{d} \phi \int_{-1}^{1} \mathrm{~d}(\cos \theta)
$$

Eq. (6) becomes with the probability (7)

$$
\begin{aligned}
P(\boldsymbol{\beta})= & \frac{1}{(2 \pi)^{3} c^{3}} \int_{0}^{\infty} \mathrm{d} \omega \omega^{2} \int_{0}^{2 \pi} \mathrm{d} \phi \int_{-1}^{1} \mathrm{~d}(\cos \theta) \\
& \times \int \frac{\mathrm{d}^{3} \boldsymbol{k}_{\mathrm{L}}}{(2 \pi)^{3}} \hbar \omega \omega\left(\beta, \boldsymbol{k}, \boldsymbol{k}_{\mathrm{L}}\right) N_{\mathrm{L}}\left(\boldsymbol{k}_{\mathrm{L}}\right) \\
= & \frac{\hbar \omega_{\mathrm{p}} \sigma_{\mathrm{T}} c\left(1-\beta^{2}\right)}{3 \pi^{4}} \int_{0}^{2 \pi} \mathrm{d} \phi \\
& \times \int_{-1}^{1} \mathrm{~d}(\cos \theta) \int_{0}^{\infty} \mathrm{d} \omega \int \mathrm{d}^{3} \boldsymbol{k}_{\mathrm{L}} N_{\mathrm{L}}\left(\boldsymbol{k}_{\mathrm{L}}\right)\left[[1-\boldsymbol{\beta} \cdot \boldsymbol{\kappa}]^{2}\right. \\
& \left.\times\left[1-\left(\boldsymbol{\beta} \cdot \boldsymbol{\kappa}_{\mathrm{L}}\right)^{2}\right]-\left(1-\beta^{2}\right)\left[\boldsymbol{\kappa} \cdot \boldsymbol{\kappa}_{\mathrm{L}}-\boldsymbol{\beta} \cdot \boldsymbol{\kappa}_{\mathrm{L}}\right]^{2}\right] \\
& \times \frac{\delta\left(\omega[1-\boldsymbol{\beta} \cdot \boldsymbol{\kappa}]-\omega_{\mathrm{p}}\left[1-\frac{k_{\mathrm{L}} c}{\omega_{\mathrm{p}}} \boldsymbol{\beta} \cdot \boldsymbol{\kappa}_{\mathrm{L}}\right]\right)}{[1-\boldsymbol{\beta} \cdot \boldsymbol{\kappa}]^{4}} .
\end{aligned}
$$

The differential spectral power of electrostatic bremsstrahlung $p(\omega, \theta, \phi, \boldsymbol{\beta})$ is defined through

$P(\boldsymbol{\beta})=\int_{0}^{2 \pi} \mathrm{d} \phi \int_{-1}^{1} \mathrm{~d}(\cos \theta) \int_{0}^{\infty} \mathrm{d} \omega p(\omega, \theta, \phi, \boldsymbol{\beta})$.

The equality of Eqs. (10) and (11) then yields

$$
\begin{aligned}
& p(\omega, \theta, \phi, \boldsymbol{\beta})=\frac{\hbar \omega_{\mathrm{p}} \sigma_{\mathrm{T}} c\left(1-\beta^{2}\right)}{3 \pi^{4}} \int_{0}^{2 \pi} \mathrm{d} \phi_{\mathrm{L}} \int_{-1}^{1} \mathrm{~d}\left(\cos \theta_{\mathrm{L}}\right) \\
& \times \int_{0}^{\infty} \mathrm{d} k_{\mathrm{L}} k_{\mathrm{L}}^{2} N_{\mathrm{L}}\left(\boldsymbol{k}_{\mathrm{L}}\right) \frac{\delta\left(\omega[1-\boldsymbol{\beta} \cdot \boldsymbol{\kappa}]-\omega_{\mathrm{p}}\left[1-\frac{k_{\mathrm{L}} c}{\omega_{\mathrm{p}}} \boldsymbol{\beta} \cdot \boldsymbol{\kappa}_{\mathrm{L}}\right]\right)}{[1-\boldsymbol{\beta} \cdot \boldsymbol{\kappa}]^{4}} \\
& \times\left[[1-\boldsymbol{\beta} \cdot \boldsymbol{\kappa}]^{2}\left[1-\left(\boldsymbol{\beta} \cdot \boldsymbol{\kappa}_{\mathrm{L}}\right)^{2}\right]-\left(1-\beta^{2}\right)\left[\boldsymbol{\kappa} \cdot \boldsymbol{\kappa}_{\mathrm{L}}-\boldsymbol{\beta} \cdot \boldsymbol{\kappa}_{\mathrm{L}}\right]^{2}\right] .
\end{aligned}
$$

Integrating over all photon angles yields the angle-integrated differential photon power

$$
\tilde{p}(\omega, \beta)=\int_{0}^{2 \pi} \mathrm{d} \phi \int_{-1}^{1} \mathrm{~d}(\cos \theta) p(\omega, \theta, \phi, \boldsymbol{\beta})
$$

\subsection{Monochromatic approximation}

We construct the monochromatic approximation of the angle integrated differential spectral power (13) as

$\tilde{p}_{\text {monochromatic }}(\omega, \boldsymbol{\beta})=P(\boldsymbol{\beta}) \delta(\omega-<\omega>)$

with the total power (11) and the mean radiated photon frequency $\langle\omega\rangle$. This mean photon energy is calculated from the zeroth and first moments of the angle-integrated differential photon power (12):

$<\omega>=\frac{S(\boldsymbol{\beta})}{P(\boldsymbol{\beta})}$

where

$$
\begin{aligned}
S(\boldsymbol{\beta}) & =\int_{0}^{\infty} \mathrm{d} \omega \omega \tilde{p}(\omega, \beta) \\
& =\int_{0}^{2 \pi} \mathrm{d} \phi \int_{-1}^{1} \mathrm{~d}(\cos \theta) \int_{0}^{\infty} \mathrm{d} \omega \omega p(\omega, \theta, \phi, \beta)
\end{aligned}
$$

and, of course,

$P(\boldsymbol{\beta})=\int_{0}^{\infty} \mathrm{d} \omega \tilde{p}(\omega, \beta)$

which is identical to Eq. (11). We note that our calculation of the mean radiated photon frequency (15) differs from the one of Melrose (1971) who has chosen different moments.

\subsection{Interlude}

Both the background plasma and the swept-up interstellar particles, are gyrotropic and isotropic in the co-moving jet frame, so that we restrict our analysis to isotropic and gyrotropic electron distribution functions. Because the spontaneous emission probability (7) involves only the relative angles between electrostatic waves, electrons and photons, respectively, there exists some freedom in chosing the azimuth $\left(\phi_{\mathrm{i}}\right)$ and pitch angles $\left(\theta_{\mathrm{i}}\right)$ of the interacting particles.

\subsubsection{Photon angle dependent quantities}

For a calculation of the angle-dependent differential spectral power (12), and thus the beaming pattern of the scattered photon, all pitch angles $\theta_{\mathrm{i}}$ are defined with respect to the direction of the ordered magnetic field $\boldsymbol{B}_{0}=\left(0,0, B_{0}\right)$, and the azimuth angles $\phi_{\mathrm{i}}$ lie in the plane perpendicular to $\boldsymbol{B}_{0}$. Because we are considering gyrotropic radiating electrons, we may set, without loss of generality, the electron's azimuth angle $\phi_{\mathrm{e}}=0$, i.e.

$\boldsymbol{\beta}=\beta\left(\sin \theta_{\mathrm{e}}, 0, \cos \theta_{\mathrm{e}}\right)$.

For the photons we chose

$\kappa=(\sin \theta \cos \phi, \sin \theta \sin \phi, \cos \theta)$.

According Eqs. (S-65)-(S-69) the electrostatic plasma waves are backward moving $\left(\theta_{\mathrm{L}}=\pi\right.$ corresponding to $\mu_{\mathrm{L}}=\cos \theta_{\mathrm{L}}=$ $-1)$. We therefore have

$\kappa_{\mathrm{L}}=(0,0,-1)$. 
Moreover, all electrostatic plasma waves are subluminal $\omega_{\mathrm{p}} / k_{\mathrm{L}}<V$ with the spectral distribution

$N_{\mathrm{L}}\left(k_{\mathrm{L}}, \mu_{\mathrm{L}}\right)=\frac{\delta\left(\mu_{\mathrm{L}}+1\right)}{2 \pi} M\left(k_{\mathrm{L}}\right)$

where

$M\left(k_{\mathrm{L}}\right)=\frac{(2 \pi)^{3} n_{\mathrm{i}} m_{\mathrm{p}} c^{3}}{\hbar \omega_{\mathrm{p}}^{2} \sqrt{\Gamma^{2}-1}} \frac{H\left[k_{\mathrm{L}}-\frac{\omega_{\mathrm{p}}}{V}\right]}{k_{\mathrm{L}}^{2}\left(\frac{c^{2} k_{\mathrm{L}}^{2}}{\omega_{\mathrm{p}}^{2}}-1\right)^{2}}$

where $V=B c=c \sqrt{1-\Gamma^{-2}}$ refers to the bulk jet speed. $H[x]$ denotes the Heaviside function $(H(x)=1$ for $x \geq 0$ and $H(x)=$ 0 for $x<0$ ). Equation (22) can be obtained as follows: we insert Eq. (21) into Eq. (8) yielding

$U_{\mathrm{L}}=\frac{\hbar \omega_{\mathrm{p}}}{(2 \pi)^{3}} \int_{0}^{\infty} \mathrm{d} k_{\mathrm{L}} k_{\mathrm{L}}^{2} M_{\mathrm{L}}\left(k_{\mathrm{L}}\right)$

and equate this to Eq. (S-67)

$$
\begin{aligned}
U_{\mathrm{L}} & =\frac{(\delta E)^{2}}{4 \pi}=\frac{n_{\mathrm{i}} m_{\mathrm{p}} c^{2}}{\sqrt{\Gamma^{2}-1}} \int_{\left(1-\Gamma^{-2}\right)^{-1 / 2}}^{\infty} \frac{\mathrm{d} N}{\left(N^{2}-1\right)^{2}} \\
& =\frac{n_{\mathrm{i}} m_{\mathrm{p}} c^{3}}{\omega_{\mathrm{p}} \sqrt{\Gamma^{2}-1}} \int_{\omega_{\mathrm{p}}\left(1-\Gamma^{-2}\right)^{-1 / 2} / c}^{\infty} \frac{\mathrm{d} k_{\mathrm{L}}}{\left(\frac{c^{2} k_{\mathrm{L}}^{2}}{\omega_{\mathrm{p}}^{2}}-1\right)^{2}}
\end{aligned}
$$

where we substituted for the index of refraction $N=c k_{\mathrm{L}} / \omega_{\mathrm{p}}$ and neglected the small factor $m_{\mathrm{e}} / m_{\mathrm{p}} \ll 1$. Comparison of Eqs. (23) and (24) immediately yields Eq. (22).

\subsubsection{Photon angle integrated quantities}

For a calculation of photon angle integrated quantities, such as the electron energy loss rate and the construction of the monochromatic approximation, it is appropriate to chose the coordinates of the interacting particles as Melrose (1971) did, i.e.

$\boldsymbol{\beta}=\beta(0,0,1), \quad \boldsymbol{\kappa}=(\sin \psi \cos \eta, \sin \psi \sin \eta, \cos \psi)$,

$\kappa_{\mathrm{L}}=\left(\sin \psi_{\mathrm{L}} \cos \eta_{\mathrm{L}}, \sin \psi_{\mathrm{L}} \sin \eta_{\mathrm{L}}, \cos \psi_{\mathrm{L}}\right)$.

The target electrostatic plasma waves can then by regarded in this frame as an isotropic distribution with the distribution

$N_{\mathrm{L}}\left(k_{\mathrm{L}}\right)=\frac{2 \pi^{2} n_{\mathrm{i}} m p c^{3}}{\hbar \omega_{\mathrm{p}}^{2} \sqrt{\Gamma^{2}-1}} \frac{H\left[k_{\mathrm{L}}-\frac{\omega_{\mathrm{p}}}{V}\right]}{k_{\mathrm{L}}^{2}\left(\frac{c^{2} k_{\mathrm{L}}^{2}}{\omega_{\mathrm{p}}^{2}}-1\right)^{2}}$.

Throughout this paper we will restrict our analysis to photon angle integrated quantities, i.e. we will adopt the choice (25).

\section{Photon-angle integrated moments of the spectral power}

With the choice (25) we obtain for the two moments (11) and (16)

$P(\boldsymbol{\beta})=\int_{0}^{2 \pi} \mathrm{d} \eta \int_{-1}^{1} \mathrm{~d}(\cos \psi) \int_{0}^{\infty} \mathrm{d} \omega p(\omega, \psi, \eta, \boldsymbol{\beta})$ and

$S(\boldsymbol{\beta})=\int_{0}^{2 \pi} \mathrm{d} \eta \int_{-1}^{1} \mathrm{~d}(\cos \psi) \int_{0}^{\infty} \mathrm{d} \omega \omega \mathrm{p}(\omega, \psi, \eta, \boldsymbol{\beta})$

where according to Eq. (12)

$$
\begin{aligned}
& p(\omega, \psi, \eta, \beta)=\frac{\hbar \omega_{\mathrm{p}} \sigma_{\mathrm{T}} c\left(1-\beta^{2}\right)}{3 \pi^{4}} \int_{0}^{2 \pi} \mathrm{d} \eta_{\mathrm{L}} \\
& \times \int_{-1}^{1} \mathrm{~d}\left(\cos \psi_{\mathrm{L}}\right) \int_{0}^{\infty} \mathrm{d} k_{\mathrm{L}} k_{\mathrm{L}}^{2} N_{\mathrm{L}}\left(k_{\mathrm{L})}\right. \\
& \quad \times \frac{\delta\left(\omega[1-\beta \cos \psi]-\omega_{\mathrm{p}}\left[1-\frac{k_{\mathrm{L}} c}{\omega_{\mathrm{p}}} \beta \cos \psi_{\mathrm{L}}\right]\right)}{[1-\beta \cos \psi]^{4}} \\
& \times\left[(1-\beta \cos \psi)^{2}\left(1-\beta^{2} \cos ^{2} \psi_{\mathrm{L}}\right)-\left(1-\beta^{2}\right)\left[\beta \cos \psi_{\mathrm{L}}\right.\right. \\
& \left.\left.-\cos \psi \cos \psi_{\mathrm{L}}-\sin \psi \sin \psi_{\mathrm{L}} \cos \left(\eta-\eta_{\mathrm{L}}\right)\right]^{2}\right] .
\end{aligned}
$$

\subsection{Total power}

Inserting Eq. (29) in Eq. (27) we obtain

$$
\begin{aligned}
P(\boldsymbol{\beta}) & =\frac{\hbar \omega_{\mathrm{p}} \sigma_{\mathrm{T}} c\left(1-\beta^{2}\right)}{3 \pi^{4}} \int_{0}^{2 \pi} \mathrm{d} \eta \int_{-1}^{1} \mathrm{~d}(\cos \psi) \\
& \times \int_{0}^{2 \pi} \mathrm{d} \eta_{\mathrm{L}} \int_{-1}^{1} \mathrm{~d}\left(\cos \psi_{\mathrm{L}}\right) \int_{0}^{\infty} \mathrm{d} k_{\mathrm{L}} k_{\mathrm{L}}^{2} N_{\mathrm{L}}\left(k_{\mathrm{L}}\right) \\
& \times \int_{0}^{\infty} \mathrm{d} \omega \frac{\delta\left(\omega[1-\beta \cos \psi]-\omega_{\mathrm{p}}\left[1-\frac{k_{\mathrm{L}} c}{\omega_{\mathrm{p}}} \beta \cos \psi_{\mathrm{L}}\right]\right)}{[1-\beta \cos \psi]^{4}} \\
& \times\left[(1-\beta \cos \psi)^{2}\left(1-\beta^{2} \cos ^{2} \psi_{\mathrm{L}}\right)-\left(1-\beta^{2}\right)\left[\beta \cos \psi_{\mathrm{L}}\right.\right. \\
& \left.\left.-\cos \psi \cos \psi_{\mathrm{L}}-\sin \psi \sin \psi_{\mathrm{L}} \cos \left(\eta-\eta_{\mathrm{L}}\right)\right]^{2}\right] .
\end{aligned}
$$

The $\delta$-function gives

$\omega[1-\beta \cos \psi]=\omega_{p}-k_{\mathrm{L}} c \beta \cos \psi_{\mathrm{L}}>0$

for

$\cos \psi_{\mathrm{L}}<\frac{\omega_{\mathrm{p}}}{k_{\mathrm{L}} \beta c}$.

We then obtain

$$
\begin{aligned}
P(\boldsymbol{\beta})= & \frac{\hbar \omega_{\mathrm{p}} \sigma_{\mathrm{T}} c\left(1-\beta^{2}\right)}{3 \pi^{4}} \int_{0}^{\infty} \mathrm{d} k_{\mathrm{L}} k_{\mathrm{L}}^{2} N_{\mathrm{L}}\left(k_{\mathrm{L}}\right) \\
& \times \int_{-1}^{1} \mathrm{~d}\left(\cos \psi_{\mathrm{L}}\right) H\left[\frac{\omega_{\mathrm{p}}}{k_{\mathrm{L}} \beta c}-\cos \psi_{\mathrm{L}}\right] \\
& \times \int_{-1}^{1} \mathrm{~d}(\cos \psi) \int_{0}^{2 \pi} \mathrm{d} \eta \int_{0}^{2 \pi} \mathrm{d} \eta_{\mathrm{L}} \\
& \times\left[\frac{1-\beta^{2} \cos ^{2} \psi_{\mathrm{L}}}{(1-\beta \cos \psi)^{3}}-\frac{1-\beta^{2}}{(1-\beta \cos \psi)^{5}}\left[(\beta-\cos \psi)^{2} \cos ^{2} \psi_{\mathrm{L}}\right.\right. \\
& +\sin ^{2} \psi \sin ^{2} \psi_{\mathrm{L}} \cos ^{2}\left(\eta-\eta_{\mathrm{L}}\right) \\
& \left.\left.-2(\beta-\cos \psi) \cos \psi_{\mathrm{L}} \sin \psi \sin \psi_{\mathrm{L}} \cos \left(\eta-\eta_{\mathrm{L}}\right)\right]\right]
\end{aligned}
$$


Performing the integrations over $\eta$ and $\eta_{\mathrm{L}}$ gives

$$
\begin{aligned}
P(\boldsymbol{\beta})= & \frac{4 \hbar \omega_{\mathrm{p}} \sigma_{\mathrm{T}} c\left(1-\beta^{2}\right)}{3 \pi^{2}} \int_{0}^{\infty} \mathrm{d} k_{\mathrm{L}} k_{\mathrm{L}}^{2} N_{\mathrm{L}}\left(k_{\mathrm{L}}\right) \\
& \times \int_{-1}^{1} \mathrm{~d}\left(\cos \psi_{\mathrm{L}}\right) H\left[\frac{\omega_{\mathrm{p}}}{k_{\mathrm{L}} \beta c}-\cos \psi_{\mathrm{L}}\right] I_{1}\left(\beta, \psi_{\mathrm{L}}\right)
\end{aligned}
$$

with the $\psi$-integral

$$
\begin{aligned}
I_{1}\left(\beta, \psi_{\mathrm{L}}\right)= & \int_{-1}^{1} \mathrm{~d}(\cos \psi)\left[\frac{1-\beta^{2} \cos ^{2} \psi_{\mathrm{L}}}{(1-\beta \cos \psi)^{3}}\right. \\
& -\frac{1-\beta^{2}}{(1-\beta \cos \psi)^{5}}\left[(\beta-\cos \psi)^{2} \cos ^{2} \psi_{\mathrm{L}}\right. \\
& \left.\left.+\frac{1}{2} \sin ^{2} \psi \sin ^{2} \psi_{\mathrm{L}}\right]\right]
\end{aligned}
$$

which is calculated in Appendix A. We obtain

$$
\begin{aligned}
P(\boldsymbol{\beta})= & \frac{16 \hbar \omega_{\mathrm{p}} \sigma_{\mathrm{T}} c \gamma^{2}}{9 \pi^{2}} \int_{0}^{\infty} \mathrm{d} k_{\mathrm{L}} k_{\mathrm{L}}^{2} N_{\mathrm{L}}\left(k_{\mathrm{L}}\right) \\
& \times \int_{-1}^{U} \mathrm{~d}\left(\cos \psi_{\mathrm{L}}\right)\left(1-\beta^{2} \cos ^{2} \psi_{\mathrm{L}}\right) \\
= & \frac{16 \hbar \omega_{\mathrm{p}} \sigma_{\mathrm{T}} c \gamma^{2}}{9 \pi^{2}} \\
& \times \int_{0}^{\infty} \mathrm{d} k_{\mathrm{L}} k_{\mathrm{L}}^{2} N_{\mathrm{L}}\left(k_{\mathrm{L}}\right)\left[(1+U)-\frac{\beta^{2}}{3}\left(1+U^{3}\right)\right]
\end{aligned}
$$

where

$$
U=\min \left[1, \frac{\omega_{\mathrm{p}}}{\beta c k_{\mathrm{L}}}\right] \text {. }
$$

\subsection{First moment}

Repeating the same steps for the first-order moment (28) we derive

$$
\begin{aligned}
S(\boldsymbol{\beta})= & \frac{\hbar \omega_{\mathrm{p}} \sigma_{\mathrm{T}} c\left(1-\beta^{2}\right)}{3 \pi^{4}} \int_{0}^{\infty} \mathrm{d} k_{\mathrm{L}} k_{\mathrm{L}}^{2} N_{\mathrm{L}}\left(k_{\mathrm{L}}\right) \\
& \times \int_{-1}^{1} \mathrm{~d}\left(\cos \psi_{\mathrm{L}}\right) H\left[\frac{\omega_{\mathrm{p}}}{k_{\mathrm{L}} \beta c}-\cos \psi_{\mathrm{L}}\right]\left(\omega_{\mathrm{p}}-k_{\mathrm{L}} \beta c \cos \psi_{\mathrm{L}}\right) \\
& \times \int_{-1}^{1} \mathrm{~d}(\cos \psi) \int_{0}^{2 \pi} \mathrm{d} \eta \int_{0}^{2 \pi} \mathrm{d} \eta_{\mathrm{L}}\left[\frac{1-\beta^{2} \cos ^{2} \psi_{\mathrm{L}}}{(1-\beta \cos \psi)^{4}}\right. \\
& -\frac{1-\beta^{2}}{(1-\beta \cos \psi)^{6}}\left[(\beta-\cos \psi)^{2} \cos ^{2} \psi_{\mathrm{L}}\right. \\
& +\sin ^{2} \psi \sin ^{2} \psi_{\mathrm{L}} \cos ^{2}\left(\eta-\eta_{\mathrm{L}}\right) \\
& \left.\left.-2(\beta-\cos \psi) \cos \psi_{\mathrm{L}} \sin \psi \sin \psi_{\mathrm{L}} \cos \left(\eta-\eta_{\mathrm{L}}\right)\right]\right]
\end{aligned}
$$

Performing again the integrations over $\eta$ and $\eta_{\mathrm{L}}$ gives

$$
\begin{aligned}
S(\boldsymbol{\beta})= & \frac{4 \hbar \omega_{\mathrm{p}} \sigma_{\mathrm{T}} c\left(1-\beta^{2}\right)}{3 \pi^{2}} \int_{0}^{\infty} \mathrm{d} k_{\mathrm{L}} k_{\mathrm{L}}^{2} N_{\mathrm{L}}\left(k_{\mathrm{L}}\right) \\
& \times \int_{-1}^{1} \mathrm{~d}\left(\cos \psi_{\mathrm{L}}\right) H\left[\frac{\omega_{\mathrm{p}}}{k_{\mathrm{L}} \beta c}-\cos \psi_{\mathrm{L}}\right]\left(\omega_{\mathrm{p}}\right. \\
& \left.-k_{\mathrm{L}} \beta c \cos \psi_{\mathrm{L}}\right) I_{2}\left(\beta, \psi_{\mathrm{L}}\right)
\end{aligned}
$$

with the $\psi$-integral

$$
\begin{aligned}
I_{2}\left(\beta, \psi_{\mathrm{L}}\right)= & \int_{-1}^{1} \mathrm{~d}(\cos \psi)\left[\frac{1-\beta^{2} \cos ^{2} \psi_{\mathrm{L}}}{(1-\beta \cos \psi)^{4}}\right. \\
& -\frac{1-\beta^{2}}{(1-\beta \cos \psi)^{6}}\left[(\beta-\cos \psi)^{2} \cos ^{2} \psi_{\mathrm{L}}\right. \\
& \left.\left.+\frac{1}{2} \sin ^{2} \psi \sin ^{2} \psi_{\mathrm{L}}\right]\right] .
\end{aligned}
$$

This integral is very similar to the integral (35) and is also calculated in Appendix A. We find

$$
\begin{aligned}
S(\boldsymbol{\beta})= & \frac{16 \hbar \omega_{\mathrm{p}}^{2} \sigma_{\mathrm{T}} c \gamma^{4}}{45 \pi^{2}} \int_{0}^{\infty} \mathrm{d} k_{\mathrm{L}} k_{\mathrm{L}}^{2} N_{\mathrm{L}}\left(k_{\mathrm{L}}\right) \\
& \times\left(\left(5+2 \beta^{2}\right)(1+U)-\left(5+2 \beta^{2}\right) \frac{\beta c k_{\mathrm{L}}}{2 \omega_{\mathrm{p}}}\left(U^{2}-1\right)\right. \\
& \left.-\frac{\beta^{2}\left(6+\beta^{2}\right)}{3}\left(1+U^{3}\right)+\beta^{2}\left(6+\beta^{2}\right) \frac{\beta c k_{\mathrm{L}}}{4 \omega_{\mathrm{p}}}\left(U^{4}-1\right)\right) .
\end{aligned}
$$

\subsection{Superluminal waves}

For superluminal $\left(\omega_{\mathrm{p}} / k_{\mathrm{L}}>c\right)$ electrostatic plasma waves, the quantity $U=1$. In this case Eq. (36) reduces to

$$
\begin{aligned}
P_{\mathrm{sl}}(\boldsymbol{\beta}) & =\frac{32 \hbar \omega_{\mathrm{p}} \sigma_{\mathrm{T}} c \gamma^{2}}{9 \pi^{2}}\left(1-\frac{\beta^{2}}{3}\right) \int_{0}^{\infty} \mathrm{d} k_{\mathrm{L}} k_{\mathrm{L}}^{2} N_{\mathrm{L}}\left(k_{\mathrm{L}}\right) \\
& =\frac{64 \sigma_{\mathrm{T}} c U_{\mathrm{L}} \gamma^{2}}{9}\left(1-\frac{\beta^{2}}{3}\right)
\end{aligned}
$$

where we used Eq. (8). Equation (41) reduces to

$$
S_{\mathrm{sl}}(\boldsymbol{\beta})=\frac{64}{9} \omega_{\mathrm{p}} \sigma_{\mathrm{T}} c \gamma^{4} U_{\mathrm{L}}\left[1-\frac{\beta^{4}}{15}\right] \text {, }
$$

so that the mean photon energy (15) in this case becomes

$$
\begin{aligned}
<\omega>_{\mathrm{s} 1} & =\frac{S_{\mathrm{sl}}}{P_{\mathrm{sl}}}=\frac{15-\beta^{4}}{5\left(3-\beta^{2}\right)} \omega_{\mathrm{p}} \gamma^{2} \\
& \simeq\left\{\begin{array}{ll}
\omega_{\mathrm{p}}\left(1+\frac{4 \beta^{2}}{3}\right) & \text { for } \beta \ll 1 \\
\frac{7}{5} \omega_{\mathrm{p}} \gamma^{2} & \text { for } \gamma>>1
\end{array} .\right.
\end{aligned}
$$

The monochromatic approximation for the angle integrated differential spectral power (14) for relativistic particles then is

$\tilde{p}_{\text {monochromatic,superluminal }}(\omega, \boldsymbol{\beta}) \simeq \frac{128}{27} \sigma_{\mathrm{T}} c U_{\mathrm{L}} \gamma^{2} \delta\left(\omega-\frac{7}{5} \omega_{\mathrm{p}} \gamma^{2}\right)$. 


\section{Monochromatic approximation for the special pick-up subluminal distribution}

In this section we calculate the photon angle integrated total power and rate of electrostatic bremsstrahlung photons for the special subluminal plasma wave distribution (26).

\subsection{Total power}

The total power (36) in this case becomes with the bulk speed of the outflow $V=B c$

$$
\begin{aligned}
P(\boldsymbol{\beta})= & \frac{32 \sigma_{\mathrm{T}} c \gamma^{2}}{9} \frac{n_{\mathrm{i}} m_{\mathrm{p}} c^{3}}{\omega_{\mathrm{p}} \sqrt{\Gamma^{2}-1}} \int_{\omega_{\mathrm{p}} / V}^{\infty} \mathrm{d} k_{\mathrm{L}}\left[\frac{c^{2} k_{\mathrm{L}}^{2}}{\omega_{\mathrm{p}}^{2}}-1\right]^{-2} \\
& \times\left[(1+U)-\frac{\beta^{2}}{3}\left(1+U^{3}\right)\right]=\frac{32 \sigma_{\mathrm{T}} c \gamma^{2}}{9} \frac{n_{\mathrm{i}} m_{\mathrm{p}} c^{2}}{\sqrt{\Gamma^{2}-1}} \\
& \times \int_{1 / B}^{\infty} \frac{\mathrm{d} x}{\left(x^{2}-1\right)^{2}}\left[(1+U)-\frac{\beta^{2}}{3}\left(1+U^{3}\right)\right]
\end{aligned}
$$

where

$U=\min \left[1, \frac{1}{\beta x}\right]=\left\{\begin{array}{ll}1 & \text { for } x \leq \frac{1}{\beta} \\ \frac{1}{\beta x} & \text { for } x>\frac{1}{\beta}\end{array}\right.$.

In the case $\beta<B$ we obtain

$$
\begin{aligned}
P(\beta<B)= & \frac{32 \sigma_{\mathrm{T}} c \gamma^{2}}{9} \frac{n_{\mathrm{i}} m_{\mathrm{p}} c^{2}}{\sqrt{\Gamma^{2}-1}}\left[2\left(1-\frac{\beta^{2}}{3}\right) \int_{1 / B}^{1 / \beta} \frac{\mathrm{d} x}{\left(x^{2}-1\right)^{2}}\right. \\
& +\left(1-\frac{\beta^{2}}{3}\right) \int_{1 / \beta}^{\infty} \frac{\mathrm{d} x}{\left(x^{2}-1\right)^{2}}+\frac{1}{\beta} \int_{1 / \beta}^{\infty} \frac{\mathrm{d} x}{x\left(x^{2}-1\right)^{2}} \\
& \left.-\frac{1}{3 \beta} \int_{1 / \beta}^{\infty} \frac{\mathrm{d} x}{x^{3}\left(x^{2}-1\right)^{2}}\right]
\end{aligned}
$$

With the integrals (2.17) of Gradshteyn \& Ryzhik (1965) we derive after straightforward but tedious algebra

$$
\begin{aligned}
P(\beta<B)= & \frac{32 \sigma_{\mathrm{T}} c \gamma^{2}}{9} \frac{n_{\mathrm{i}} m_{\mathrm{p}} c^{2}}{\sqrt{\Gamma^{2}-1}}\left[( 1 - \frac { \beta ^ { 2 } } { 3 } ) \left[\Gamma \sqrt{\Gamma^{2}-1}\right.\right. \\
& \left.-\ln ((1+B) \Gamma)-\frac{1}{2}\left(\beta \gamma^{2}-\ln ((1+\beta) \gamma)\right)\right] \\
& \left.+\frac{\beta}{6}\left(1+\beta^{2}\right) \gamma^{2}-\frac{\ln \gamma}{3 \beta}\right] \\
\simeq & \frac{32}{9} \sigma_{\mathrm{T}} c \gamma^{2}\left(1-\frac{\beta^{2}}{3}\right) \Gamma n_{\mathrm{i}} m_{\mathrm{p}} c^{2} \\
= & \frac{64}{9} \sigma_{\mathrm{T}} c U_{\mathrm{L}} \gamma^{2}\left(1-\frac{\beta^{2}}{3}\right)
\end{aligned}
$$

where we have used the total energy density (1). Despite the more involved integration over the wavenumber distribution of the plasma waves, the final result (49) agrees remarkably well with the total rate (42) derived for superluminal plasma waves.
In the case $\beta \geq B$ we find

$$
\begin{aligned}
P(\beta \geq B)= & \frac{32 \sigma_{\mathrm{T}} c \gamma^{2}}{9} \frac{n_{\mathrm{i}} m_{\mathrm{p}} c^{2}}{\sqrt{\Gamma^{2}-1}}\left[\left(1-\frac{\beta^{2}}{3}\right) \int_{1 / B}^{\infty} \frac{\mathrm{d} x}{\left(x^{2}-1\right)^{2}}\right. \\
& \left.+\frac{1}{\beta} \int_{1 / B}^{\infty} \frac{\mathrm{d} x}{x\left(x^{2}-1\right)^{2}}-\frac{1}{3 \beta} \int_{1 / B}^{\infty} \frac{\mathrm{d} x}{x^{3}\left(x^{2}-1\right)^{2}}\right] \\
= & \frac{32 \sigma_{\mathrm{T}} c \gamma^{2}}{9} \frac{n_{\mathrm{i}} m_{\mathrm{p}} c^{2}}{\sqrt{\Gamma^{2}-1}}\left[\frac{1}{2}\left(1-\frac{\beta^{2}}{3}\right)\left[B \Gamma^{2}-\ln ((1+B) \Gamma)\right]\right. \\
& \left.-\frac{\ln \Gamma}{3 \beta}+\frac{B^{2} \Gamma^{2}\left(1+B^{2}\right)}{6 \beta}\right] \\
\simeq & \frac{16 \sigma_{\mathrm{T}} c \gamma^{2}}{9} \Gamma n_{\mathrm{i}} m_{\mathrm{p}} c^{2}\left[1+\frac{B}{3 \beta}-\frac{\beta^{2}}{3}\left(1-\left(\frac{B}{\beta}\right)^{3}\right)\right] \\
= & \frac{32}{9} \sigma_{\mathrm{T}} c \gamma^{2} U_{\mathrm{L}}\left[1+\frac{B}{3 \beta}-\frac{\beta^{2}}{3}\left(1-\left(\frac{B}{\beta}\right)^{3}\right)\right]
\end{aligned}
$$

which for $\beta>>B$ approaches

$P(\beta>>B) \simeq \frac{32}{9} \sigma_{\mathrm{T}} c U_{\mathrm{L}} \gamma^{2}\left(1-\frac{\beta^{2}}{3}\right)$

which is half the value of Eq. (49).

\subsection{First moment}

The first moment (41) in this case becomes

$$
\begin{aligned}
S(\beta)= & \frac{32}{45} \sigma_{\mathrm{T}} c \gamma^{4} \frac{n_{\mathrm{i}} m_{\mathrm{p}} c^{3}}{\sqrt{\Gamma^{2}-1}} \int_{\omega_{\mathrm{p}} / V}^{\infty} \mathrm{d} k_{\mathrm{L}}\left[\frac{c^{2} k_{\mathrm{L}}^{2}}{\omega_{\mathrm{p}}^{2}}-1\right]^{-2} \\
& \times\left(\left(5+2 \beta^{2}\right)(1+U)-\left(5+2 \beta^{2}\right) \frac{\beta c k_{\mathrm{L}}}{2 \omega_{\mathrm{p}}}\left(U^{2}-1\right)\right. \\
& \left.-\frac{\beta^{2}\left(6+\beta^{2}\right)}{3}\left(1+U^{3}\right)+\beta^{2}\left(6+\beta^{2}\right) \frac{\beta c k_{\mathrm{L}}}{4 \omega_{\mathrm{p}}}\left(U^{4}-1\right)\right) \\
= & \frac{32}{45} \omega_{\mathrm{p}} \sigma_{\mathrm{T}} c \gamma^{4} \frac{n_{\mathrm{i}} m_{\mathrm{p}} c^{2}}{\sqrt{\Gamma^{2}-1}} \int_{1 / B}^{\infty} \mathrm{d} x\left[x^{2}-1\right]^{-2} \\
& \times\left(\left(5+2 \beta^{2}\right)(1+U)--\frac{\beta x}{2}\left(5+2 \beta^{2}\right)\left(U^{2}-1\right)\right. \\
& \left.-\frac{\beta^{2}\left(6+\beta^{2}\right)}{3}\left(1+U^{3}\right)+\frac{\beta x}{4} \beta^{2}\left(6+\beta^{2}\right)\left(U^{4}-1\right)\right) .(52)
\end{aligned}
$$

In the case $\beta<B$ we obtain

$$
\begin{aligned}
S(\beta<B)= & \frac{32}{45} \omega_{\mathrm{p}} \sigma_{\mathrm{T}} c \gamma^{4} \frac{n_{\mathrm{i}} m_{\mathrm{p}} c^{2}}{\sqrt{\Gamma^{2}-1}} \\
& \times\left[2\left[5+2 \beta^{2}-\frac{\beta^{2}\left(6+\beta^{2}\right)}{3}\right] \int_{1 / B}^{1 / \beta} \mathrm{d} x\left[x^{2}-1\right]^{-2}\right. \\
& +\int_{1 / \beta}^{\infty} \mathrm{d} x\left[x^{2}-1\right]^{-2}\left(\left(5+2 \beta^{2}\right)\left(1+\frac{1}{\beta x}\right)\right. \\
& -\frac{\beta x}{2}\left(5+2 \beta^{2}\right)\left(\frac{1}{\beta^{2} x^{2}}-1\right)-\frac{\beta^{2}\left(6+\beta^{2}\right)}{3} \\
& \left.\left.\times\left(1+\frac{1}{\beta^{3} x^{3}}\right)+\frac{\beta x}{4} \beta^{2}\left(6+\beta^{2}\right)\left(\frac{1}{\beta^{4} x^{4}}-1\right)\right)\right]
\end{aligned}
$$




$$
\begin{aligned}
= & \frac{32}{45} \omega_{\mathrm{p}} \sigma_{\mathrm{T}} c \gamma^{4} \frac{n_{\mathrm{i}} m_{\mathrm{p}} c^{2}}{\sqrt{\Gamma^{2}-1}}\left[\left[5+2 \beta^{2}-\frac{\beta^{2}\left(6+\beta^{2}\right)}{3}\right]\right. \\
& \times\left[B \Gamma^{2}-\ln ((1+B) \Gamma)\right] \\
& -\frac{1}{2}\left[5+2 \beta^{2}-\frac{\beta^{2}\left(6+\beta^{2}\right)}{3}\right] \\
& \times\left[\beta \gamma^{2}-\ln ((1+\beta) \gamma)\right] \\
& \left.+\frac{\beta \gamma^{2}}{24}\left(18+46 \beta^{2}-5 \beta^{4}-3 \beta^{6}\right)-\frac{9+5 \beta^{2}}{6 \beta} \ln \gamma\right] \\
\simeq & \frac{32}{45} \hbar \omega_{\mathrm{p}}^{2} \sigma_{\mathrm{T}} c \gamma^{4} \Gamma n_{\mathrm{i}} m_{\mathrm{p}} c^{2}\left[5-\frac{\beta^{4}}{3}\right] \\
= & \frac{64}{9} \omega_{\mathrm{p}} \sigma_{\mathrm{T}} c U_{\mathrm{L}} \gamma^{4}\left[1-\frac{\beta^{4}}{15}\right] .
\end{aligned}
$$

In the case $\beta \geq B$ we obtain

$$
\begin{aligned}
S(\beta \geq B)= & \frac{32}{45} \omega_{\mathrm{p}} \sigma_{\mathrm{T}} c \gamma^{4} \frac{n_{\mathrm{i}} m_{\mathrm{p}} c^{2}}{\sqrt{\Gamma^{2}-1}} \int_{1 / B}^{\infty} \mathrm{d} x\left[x^{2}-1\right]^{-2} \\
& \times\left(\left(5+2 \beta^{2}\right)\left(1+\frac{1}{\beta x}\right)-\frac{\beta x}{2}\left(5+2 \beta^{2}\right)\left(\frac{1}{\beta^{2} x^{2}}-1\right)\right. \\
& -\frac{\beta^{2}\left(6+\beta^{2}\right)}{3}\left(1+\frac{1}{\beta^{3} x^{3}}\right)+\frac{\beta x}{4} \beta^{2}\left(6+\beta^{2}\right) \\
& \left.\times\left(\frac{1}{\beta^{4} x^{4}}-1\right)\right) \\
= & \frac{32}{45} \omega_{\mathrm{p}} \sigma_{\mathrm{T}} c \gamma^{4} \frac{n_{\mathrm{i}} m_{\mathrm{p}} c^{2}}{\sqrt{\Gamma^{2}-1}} \\
& \times\left[\frac{1}{2}\left[5+2 \beta^{2}-\frac{\beta^{2}\left(6+\beta^{2}\right)}{3}\right]\left[B \Gamma^{2}-\ln ((1+B) \Gamma)\right]\right. \\
& -\frac{9+5 \beta^{2}}{6 \beta} \ln \Gamma+\frac{9+5 \beta^{2}}{12 \beta} B^{2} \Gamma^{2} \\
& \left.+\frac{6+\beta^{2}}{24 \beta} B^{4} \Gamma^{2}+\frac{\beta B^{2} \Gamma^{2}}{8}\left[10-2 \beta^{2}-\beta^{4}\right] \frac{1}{2}\right] . \quad(54)
\end{aligned}
$$

In the following we limit our analysis to the case $\beta<B$.

\subsection{Monochromatic approximation}

With Eqs. (49) and (53) we obtain for the mean photon energy (15) in this subluminal case

$<\omega>_{\text {sub }}=\frac{15-\beta^{4}}{5\left(3-\beta^{2}\right)} \omega_{\mathrm{p}} \gamma^{2} \simeq \begin{cases}\omega_{\mathrm{p}}\left(1+\frac{4 \beta^{2}}{3}\right) & \text { for } \beta \ll 1 \\ \frac{7}{5} \omega_{\mathrm{p}} \gamma^{2} & \text { for } \gamma>>1\end{cases}$

which is identical to the corresponding expression (44) in the superluminal case.

The monochromatic approximation for the angle integrated differential spectral power (14) then is

$\tilde{p}_{\text {monochromatic,subluminal }}(\omega, \boldsymbol{\beta}) \simeq$

$$
\frac{64}{9} \sigma_{\mathrm{T}} c U_{\mathrm{L}} \gamma^{2}\left(1-\frac{\beta^{2}}{3}\right) \delta\left(\omega-\frac{15-\beta^{4}}{5\left(3-\beta^{2}\right)} \omega_{\mathrm{p}} \gamma^{2}\right)
$$

which for relativistic particles is identical to the corresponding expression (45) in the superluminal case.

With $\omega=2 \pi v$ we obtain for the monochromatic approximation for the photon-angle integrated differential spectral power per frequency

$\tilde{p}_{\text {monochromatic }}(v, \beta) \simeq$

$\frac{64}{9} \sigma_{\mathrm{T}} c U_{\mathrm{L}} \gamma^{2}\left(1-\frac{\beta^{2}}{3}\right) \delta\left(v-\frac{15-\beta^{4}}{5\left(3-\beta^{2}\right)} v_{\mathrm{p}} \gamma^{2}\right) \mathrm{erg} \mathrm{s}^{-1} \mathrm{~Hz}^{-1}$

where

$v_{\mathrm{p}}=\omega_{\mathrm{p}} / 2 \pi=1.3 \times 10^{8} n_{\mathrm{b}, 8}^{1 / 2} \quad \mathrm{~Hz}$

with the co-moving pair density $n_{\mathrm{b}, 8}=n_{\mathrm{b}, 10}^{*} / \Gamma_{2}$ in units of $10^{8} \mathrm{~cm}^{-3}$.

\section{Bulk pair electrostatic bremsstrahlung}

As our first application we consider the electrostatic bremsstrahlung radiated by the thermal pair background plasma in the comoving outflow plasma. These pairs have the comoving thermal isotropic distribution function

$N_{\mathrm{b}}(p)=\frac{n_{\mathrm{b}} p^{2}}{\left(m_{\mathrm{e}} c\right)^{3} \Theta K_{2}\left(\Theta^{-1}\right)} \exp \left[-\frac{\sqrt{1+\frac{p^{2}}{m_{\mathrm{e}}^{2} c^{2}}}}{\Theta}\right]$

characterised by the total density $n_{\mathrm{b}}$ and the dimensionless pair temperature $\Theta=k_{\mathrm{B}} T_{\mathrm{e}} /\left(m_{\mathrm{e}} c^{2}\right) . K_{2}$ denotes the modified Bessel function of second order. For non-relativistic $(\Theta \ll 1)$ temperatures the distribution (59) is well approximated by

$$
\begin{aligned}
N_{\mathrm{b}}(p) & \simeq \sqrt{\frac{2}{\pi}} \frac{p^{2} n_{\mathrm{b}}}{\Theta^{3 / 2} m_{\mathrm{e}}^{3} c^{3}} \exp \left[-\frac{p^{2}}{2 \Theta m_{\mathrm{e}}^{2} c^{2}}\right] \\
& =\sqrt{\frac{2}{\pi}} \frac{n_{\mathrm{b}} \beta^{2}}{\Theta^{3 / 2} m_{\mathrm{e}} c} \exp \left[-\frac{\beta^{2}}{2 \Theta}\right] .
\end{aligned}
$$

The spontaneous emission coefficient of electrostatic bremsstrahlung from the bulk pairs is given by (Bekefi 1966)

$j(v)=\frac{1}{4 \pi} \int_{0}^{\infty} \mathrm{d} p N_{\mathrm{b}}\left((p) \tilde{p}_{\text {monochromatic }}(\nu, \beta(p))\right.$

$\operatorname{erg~s}{ }^{-1} \mathrm{~Hz}^{-1}$ ster $^{-1} \mathrm{~cm}^{-3}$

whereas the corresponding absorption coefficient is

$\alpha(v)=-\frac{1}{2 n_{\mathrm{r}}^{2} v^{2}} \int_{0}^{\infty} \mathrm{d} p p \sqrt{p^{2} c^{2}+m_{\mathrm{e}}^{2} c^{4}}$

$\tilde{p}_{\text {monochromatic }}(\nu, \beta(p)) \frac{\partial}{\partial p}\left(\frac{N_{\mathrm{b}}(p)}{p^{2}}\right) \quad \mathrm{cm}^{-1}$

with the index of refraction

$n_{\mathrm{r}}^{2}=1-\frac{v_{\mathrm{p}}^{2}}{v^{2}}$ 


\subsection{Spontaneous emission}

With Eqs. (57) and (60) the spontaneous emission coefficient (61) for non-relativistic pair temperatures becomes

$$
\begin{aligned}
j(v) \simeq & \frac{16 \sqrt{2}}{9 \pi^{3 / 2}} \frac{\sigma_{\mathrm{T}} c U_{\mathrm{L}} n_{\mathrm{b}}}{\Theta^{3 / 2}} \\
& \times \int_{0}^{\infty} \mathrm{d} \beta \beta^{2}\left(1+\frac{2}{3} \beta^{2}\right) \delta\left(v-v_{\mathrm{p}}\left(1+\frac{4}{3} \beta^{2}\right)\right) \exp \left(-\frac{\beta^{2}}{2 \Theta}\right) \\
= & \frac{2^{3 / 2}}{3 \pi^{3 / 2}} \frac{\sigma_{\mathrm{T}} c U_{\mathrm{L}} n_{\mathrm{b}}}{v_{\mathrm{p}} \Theta^{3 / 2}} \\
& \times \int_{0}^{\infty} \mathrm{d} \beta \beta\left(1+\frac{2}{3} \beta^{2}\right) \delta\left(\beta-\beta_{0}\right) \exp \left(-\frac{\beta^{2}}{2 \Theta}\right)
\end{aligned}
$$

where

$\beta_{0}=\sqrt{\frac{3\left(v-v_{\mathrm{p}}\right)}{4 v_{\mathrm{p}}}}$.

We obtain

$$
\begin{aligned}
j(v)= & \frac{1}{6^{1 / 2} \pi^{3 / 2}} \frac{\sigma_{\mathrm{T}} c U_{\mathrm{L}} n_{\mathrm{b}}}{v_{\mathrm{p}} \Theta^{3 / 2}} H\left[v-v_{\mathrm{p}}\right]\left[\frac{v}{v_{\mathrm{p}}}-1\right]^{1 / 2} \\
& \times\left(\frac{v}{v_{\mathrm{p}}}+1\right) \exp \left(-\frac{3\left(v-v_{\mathrm{p}}\right)}{8 \Theta v_{\mathrm{p}}}\right)
\end{aligned}
$$

which attains its maximum value near $v_{\mathrm{m}}=v_{\mathrm{p}}(1+(4 \Theta / 3))$.

\subsection{Absorption coefficient}

With Eqs. (57) and (60) the absorption coefficient (62) for nonrelativistic pair temperatures becomes

$$
\begin{aligned}
\alpha(v) \simeq & \frac{64}{9 \sqrt{2 \pi}} \frac{\sigma_{\mathrm{T}} c U_{\mathrm{L}} n_{\mathrm{b}}}{\Theta^{5 / 2} m_{\mathrm{e}}\left(v^{2}-v_{\mathrm{p}}^{2}\right)} \\
& \times \int_{0}^{\infty} \mathrm{d} \beta \beta^{2}\left(1+\frac{2}{3} \beta^{2}\right) \delta\left(v-v_{\mathrm{p}}\left(1+\frac{4}{3} \beta^{2}\right)\right) \exp \left(-\frac{\beta^{2}}{2 \Theta}\right) \\
= & \frac{8}{3 \sqrt{2 \pi}} \frac{\sigma_{\mathrm{T}} c U_{\mathrm{L}} n_{\mathrm{b}}}{\Theta^{5 / 2} m_{\mathrm{e}} v_{\mathrm{p}}\left(v^{2}-v_{\mathrm{p}}^{2}\right)} \\
& \times \int_{0}^{\infty} \mathrm{d} \beta \beta\left(1+\frac{2}{3} \beta^{2}\right) \delta\left(\beta-\beta_{0}\right) \exp \left(-\frac{\beta^{2}}{2 \Theta}\right) .
\end{aligned}
$$

We obtain

$$
\alpha(v)=\sqrt{\frac{2}{3 \pi}} \frac{\sigma_{\mathrm{T}} c U_{\mathrm{L}} n_{\mathrm{b}}}{\Theta^{5 / 2} m_{\mathrm{e}} v_{\mathrm{p}}^{3}} H\left[v-v_{\mathrm{p}}\right] \frac{\exp \left(-\frac{3\left(v-v_{\mathrm{p}}\right)}{8 \Theta v_{\mathrm{p}}}\right)}{\left[\frac{v}{v_{\mathrm{p}}}-1\right]^{1 / 2}} .
$$

The optical depth for the uniform outflow region of length $L=$ $10^{15} L_{15} \mathrm{~cm}$ then is

$$
\begin{aligned}
\tau(v)= & \alpha L=\sqrt{\frac{2}{3 \pi}} \frac{\sigma_{\mathrm{T}} c U_{\mathrm{L}} n_{\mathrm{b}} L}{\Theta^{5 / 2} m_{\mathrm{e}} v_{\mathrm{p}}^{3}} H\left[v-v_{\mathrm{p}}\right]\left[\frac{v}{v_{\mathrm{p}}}-1\right]^{-1 / 2} \\
& \times \exp \left(-\frac{3\left(v-v_{\mathrm{p}}\right)}{8 \Theta v_{\mathrm{p}}}\right) \\
= & 10^{14} \Gamma_{2}^{5 / 2} n_{\mathrm{i}}^{*} L_{15}\left(n_{\mathrm{b}, 10}^{*}\right)^{-1 / 2} \\
& \times \Theta_{-1}^{-5 / 2} H\left[v-v_{\mathrm{p}}\right]\left[\frac{v}{v_{\mathrm{p}}}-1\right]^{-1 / 2} \exp \left(-\frac{3\left(v-v_{\mathrm{p}}\right)}{8 \Theta v_{\mathrm{p}}}\right) .
\end{aligned}
$$

The numerical value of the optical depth is calculated for a dimensionless pair temperature value of $\Theta=0.1 \Theta_{-1}$.

The outflow region is optically thin for values of $\tau \leq 1$ and optically thick for values of $\tau>1$. According to Eq. (69) this corresponds to the frequency ranges $v \geq v_{\mathrm{c}}$ and $v_{\mathrm{p}} \leq v<v_{\mathrm{c}}$, respectively, where the characteristic frequency is given by $\tau\left(v_{\mathrm{c}}\right)=1$

$$
\begin{aligned}
v_{\mathrm{c}} \simeq & v_{\mathrm{p}}\left(1+\frac{8 \Theta}{3} \ln \left(\sqrt{\frac{2}{3 \pi}} \frac{\sigma_{\mathrm{T}} c U_{\mathrm{L}} n_{\mathrm{b}} L}{\Theta^{5 / 2} m_{\mathrm{e}} \nu_{\mathrm{p}}^{3}}\right)\right) \\
= & v_{\mathrm{p}}\left(1+\frac{4 \Theta_{-1}}{15} \ln \left[10^{14} \Gamma_{2}^{5 / 2} n_{\mathrm{i}}^{*} L_{15}\left(n_{\mathrm{b}, 10}^{*}\right)^{-1 / 2} \Theta_{-1}^{-5 / 2}\right]\right) \\
= & v_{\mathrm{p}}\left(1+8.6 \Theta_{-1}\left[1+0.108 \ln \Gamma_{2}\right.\right. \\
& \left.\left.-0.077 \ln \Theta-1+0.03 \ln \frac{n_{\mathrm{i}}^{*} d_{13}^{*}}{n_{\mathrm{b}, 10}^{*}}\right]\right)
\end{aligned}
$$

where we assumed the length scale $L_{15}=d_{15}=d_{13}^{*} \Gamma_{2}$ to equal the thickness of the outflow region.

\subsection{Intensity of bulk pair electrostatic bremsstrahlung}

In the optically thick frequency range $v_{\mathrm{p}} \leq v \leq v_{\mathrm{c}}$ the intensity of bulk pair electrostatic bremsstrahlung is

$I\left(v_{\mathrm{p}} \leq v \leq v_{\mathrm{c}}\right) \simeq \frac{j(v)}{n_{\mathrm{r}}^{2} \alpha(v)}=\frac{m_{\mathrm{e}} \Theta}{2 \pi} v^{2}$

$=\frac{v^{2}}{2 \pi c^{2}} k_{\mathrm{B}} T_{\mathrm{e}} \quad \operatorname{erg~s}^{-1} \mathrm{~Hz}^{-1} \operatorname{ster}^{-1} \mathrm{~cm}^{-2}$

whereas in the optically thin regime

$$
\begin{aligned}
I\left(v>v_{\mathrm{c}}\right) \simeq & j\left(v>v_{\mathrm{c}}\right) L=\frac{1}{6^{1 / 2} \pi^{3 / 2}} \frac{\sigma_{\mathrm{T}} c U_{\mathrm{L}} n_{\mathrm{b}} L}{v_{\mathrm{p}} \Theta^{3 / 2}} H\left[v-v_{\mathrm{p}}\right] \\
& \times\left[\frac{v}{v_{\mathrm{p}}}-1\right]^{1 / 2}\left(\frac{v}{v_{\mathrm{p}}}+1\right) \exp \left(-\frac{3\left(v-v_{\mathrm{p}}\right)}{8 \Theta v_{\mathrm{p}}}\right) \\
& \operatorname{erg~s}^{-1} \mathrm{~Hz}^{-1} \mathrm{ster}^{-1} \mathrm{~cm}^{-2} .
\end{aligned}
$$

\section{Energy loss rate and relativistic electron equilibrium energy spectrum}

Before we consider the electrostatic bremsstrahlung from the swept-up interstellar electrons, we have to determine the equilibrium spectrum of these electrons which results from the balance of injection, escape and energy loss processes in the outflow region.

According to Sect. 4 of Schlickeiser et al. (2002), after pitch-angle isotropisation, operating on the co-moving time scale (Eq. (S-133))

$T_{\text {iso }}=0.19 \frac{\left(n_{\mathrm{b}, 10}^{*}\right)^{1 / 2}}{n_{\mathrm{i}}^{*} \Gamma_{2}^{1 / 6}} \mathrm{~s}$,

the swept-up interstellar electrons attain the flat and isotropic differential volume-integrated injection spectrum per unit Lorentz factor

$Q_{\mathrm{e}}(\gamma)=c \sqrt{\Gamma^{2}-1} n_{\mathrm{i}}^{*} \pi R^{2} H[\Gamma-\gamma] \quad \mathrm{s}^{-1}$ 
where $P=\Gamma m_{\mathrm{e}} V=\Gamma m_{\mathrm{e}} B c=m_{\mathrm{e}} c \sqrt{\Gamma^{2}-1} \simeq m_{\mathrm{e}} c \Gamma=$ $51 \Gamma_{2} \mathrm{MeV} / \mathrm{c}$.

After injection, these electrons suffer continuous energy losses in the pair background plasma due to Møller and Bhabha scattering and $\mathrm{e}^{-} \mathrm{e}^{-}$and $\mathrm{e}^{-} \mathrm{e}^{+}$bremsstrahlung with the loss rates (Böttcher et al. 2001)

$\dot{\gamma}_{\mathrm{M} \varnothing} \simeq-2.6 \times 10^{-3} n_{\mathrm{b}, 8} \gamma^{-0.02} \mathrm{~s}^{-1}$

and

$\dot{\gamma}_{\mathrm{br}} \simeq-8.0 \times 10^{-8} n_{\mathrm{b}, 8} \gamma^{1.15} \mathrm{~s}^{-1}$.

The swept-up electrons will also emit synchrotron radiation, thus losing energy at the rate (Schlickeiser \& Crusius 1988)

$$
\begin{aligned}
\dot{\gamma}_{\mathrm{sy}} & =-2 \frac{c \sigma_{\mathrm{T}}}{m_{\mathrm{e}} c^{2}} U_{\mathrm{B}} \gamma^{2} \exp \left(-\gamma_{\mathrm{R}} / \gamma\right) \\
& =1.3 \times 10^{-9}\left(B_{0} / \text { Gauss }\right)^{2} \gamma^{2} \exp \left(-\gamma_{\mathrm{R}} / \gamma\right) \mathrm{s}^{-1}
\end{aligned}
$$

where $U_{\mathrm{B}}=B_{0}^{2} / 8 \pi=0.04\left(B_{0} / \text { Gauss }\right)^{2}$ erg $\mathrm{cm}^{-3}$ denotes the magnetic energy density in the jet plasma. The RazinTsytovich Lorentz factor

$\gamma_{\mathrm{R}}=\frac{2 v_{\mathrm{p}}}{3 v_{0}}=30\left(n_{\mathrm{b}, 10}^{*}\right)^{1 / 2} \Gamma_{2}^{-1 / 2}\left(B_{0} / \text { Gauss }\right)^{-1}$,

is defined by the plasma frequency and the electron gyrofrequency $v_{0}=2.8 \times 10^{6}\left(B_{0} /\right.$ Gauss $) \mathrm{Hz}$, and accounts for the suppression of synchrotron radiation in the pair background plasma.

Moreover, the electrons lose energy by electrostatic bremsstrahlung radiation with the loss rate

$\frac{\mathrm{d} E}{\mathrm{~d} t}=m_{\mathrm{e}} c^{2} \dot{\gamma}_{\mathrm{eb}}=-P(\beta)$

given by the total power (49). For relativistic energies we obtain

$\dot{\gamma}_{\mathrm{eb}} \simeq-\frac{128}{27} \frac{c \sigma_{\mathrm{T}}}{m_{\mathrm{e}} c^{2}} U_{\mathrm{L}} \gamma^{2}$.

Comparing the electrostatic bremsstrahlung loss rate (80) with the synchrotron radiation loss rate (77) we first note that electrostatic bremsstrahlung dominates synchrotron radiation if

$U_{\mathrm{L}}>\frac{27}{64} U_{\mathrm{B}} \simeq 0.42 U_{\mathrm{B}}$

which is fulfilled for standard inner jet outflow parameters as discussed above (see Eq. (1)).

The total electron loss rate for standard jet outflow parameters is then given by

$$
\begin{aligned}
-\dot{\gamma}_{\mathrm{tot}}= & -\left(\dot{\gamma}_{\mathrm{M} \varnothing}+\dot{\gamma}_{\mathrm{br}}+\dot{\gamma}_{\mathrm{sy}}+\dot{\gamma}_{\mathrm{eb}}\right) \\
= & 2.6 \times 10^{-3} \frac{n_{\mathrm{b}, 10}^{*}}{\Gamma_{2}} \gamma^{-0.02}\left[1+\left(\frac{\gamma}{7183}\right)^{1.17}\right] \\
& +2.4 \times 10^{-6} \Gamma_{2}^{2} n_{\mathrm{i}}^{*} \gamma^{2}\left[1+5.5 \times 10^{-4}\left(\frac{B_{0}}{\text { Gauss }}\right)^{2}\right] \\
\simeq & \begin{cases}2.6 \times 10^{-3} \frac{n_{\mathrm{b}, 10}^{*}}{\Gamma_{2}} \gamma^{-0.02} & \text { for } \gamma \leq \gamma_{\mathrm{c}} \\
2.4 \times 10^{-6} \Gamma_{2}^{2} n_{\mathrm{i}}^{*} \gamma^{2} & \text { for } \gamma>\gamma_{\mathrm{c}}\end{cases}
\end{aligned}
$$

where

$\gamma_{\mathrm{c}}=\left[\frac{2.6 \times 10^{3}}{2.4} \frac{n_{\mathrm{b}, 10}^{*}}{n_{\mathrm{i}}^{*} \Gamma_{2}^{3}}\right]^{\frac{1}{2.02}} \simeq 32\left[\frac{n_{\mathrm{b}, 10}^{*}}{n_{\mathrm{i}}^{*} \Gamma_{2}^{3}}\right]^{1 / 2}$ denotes the characteristic electron Lorentz factor where the Møller and Bhabha loss rates exactly equal the electrostatic bremsstrahlung loss rate. The careful reader will note that we omitted the Razin-Tsytovich suppression factor in the total loss rate; as discussed by Schlickeiser \& Crusius (1988) this is justified because it is of minor influence as compared to Møller and Bhabha scattering losses.

For standard jet outflow parameters Møller and Bhabha scattering and electrostatic bremsstrahlung are the dominant continous energy loss processes whereas electron-electronbremsstrahlung, electron-positron-bremsstrahlung and synchrotron radiation are negligibly small in shaping the electron equilibrium electron spectrum.

From the total loss rate (82) we directly obtain the energy loss time scale as

$$
\begin{aligned}
T_{\text {loss }}(\gamma) & =\frac{\gamma}{\left|\dot{\gamma}_{\mathrm{tot}}\right|} \\
& \simeq \begin{cases}400 \frac{\Gamma_{2}}{n_{\mathrm{b}, 10}^{*}} \gamma^{1.02} & \text { for } \gamma \leq \gamma_{\mathrm{c}} \\
4 \times 10^{5} \Gamma_{2}^{-2}\left(n_{\mathrm{i}}^{*}\right)^{-1} \gamma^{-1} & \text { for } \gamma>\gamma_{\mathrm{c}}\end{cases}
\end{aligned}
$$

which attains its maximum value $T_{\text {loss, } \max }=1300 \Gamma_{2} / n_{\mathrm{b}, 10}^{*} \mathrm{~s}$ at $\gamma=\gamma_{\mathrm{c}}$.

The energy loss time scale (84) has to be compared with the escape time of the isotropised electrons from the outflow region. The escape time by spatial diffusion is given by the light crossing time scale,

$T_{1}=d / c=3 \times 10^{4} d_{13}^{*} \Gamma_{2} \quad \mathrm{~s}$,

and the isotropisation time scale (73) as

$T_{\mathrm{e}} \simeq \frac{T_{1}^{2}}{T_{\text {iso }}}=5 \times 10^{9} \frac{\left(d_{13}^{*}\right)^{2} \Gamma_{2}^{13 / 6} n_{\mathrm{i}}^{*}}{\left(n_{\mathrm{b}, 10}^{*}\right)^{1 / 2}} \mathrm{~s}$.

For standard jet outflow parameters, at all electron Lorentz factors $\gamma \leq 100 \Gamma_{2}$ the total electron energy loss time scale (84) is more than six orders of magnitude smaller than the escape time. The outflow region thus is a thick target for the swept-up interstellar electrons. In this case the volume-integrated equilibrium electron energy spectrum per unit Lorentz factor is simply given by

$$
\begin{aligned}
N_{\mathrm{e}}(\gamma)= & \frac{1}{\left|\dot{\gamma}_{\mathrm{tot}}(\gamma)\right|} \int_{\gamma}^{\infty} \mathrm{d} \gamma^{\prime} Q_{\mathrm{e}}\left(\gamma^{\prime}\right) \\
= & \pi R^{2} c \sqrt{\Gamma^{2}-1} n_{\mathrm{i}}^{*} H[\Gamma-\gamma] \frac{\gamma}{\left|\dot{\gamma}_{\mathrm{tot}}(\gamma)\right|} \\
= & \pi R^{2} c \sqrt{\Gamma^{2}-1} n_{\mathrm{i}}^{*} T_{\text {loss }}(\gamma) H[\Gamma-\gamma] \\
\simeq & \pi R^{2} c \sqrt{\Gamma^{2}-1} n_{\mathrm{i}}^{*} \\
& \times \begin{cases}400 \Gamma_{2}\left(n_{\mathrm{b}, 10}^{*}\right)^{-1} \gamma^{1.02} & \text { for } \gamma \leq \gamma_{\mathrm{c}}<\Gamma \\
4 \times 10^{5} \Gamma_{2}^{-2}\left(n_{\mathrm{i}}^{*}\right)^{-1} \gamma^{-1} & \text { for } \gamma_{\mathrm{c}}<\gamma \leq \Gamma \\
0 & \text { for } \gamma>\Gamma .\end{cases}
\end{aligned}
$$

The corresponding differential equilibrium electron density spectrum per unit Lorentz factor follows from Eq. (87) by dividing through the volume of the cylindric outflow region $\pi R^{2} d$ yielding

$n_{\mathrm{e}}(\gamma)=\frac{N_{\mathrm{e}}(\gamma)}{\pi R^{2} d}=\sqrt{\Gamma^{2}-1} n_{\mathrm{i}}^{*} \frac{T_{\mathrm{loss}}(\gamma)}{T_{1}} H[\Gamma-\gamma]$ 
which involves the light crossing time scale (85). With the notation introduced above we obtain

$n_{\mathrm{e}}(\gamma)=n_{\mathrm{e}, 0} H[\Gamma-\gamma] \frac{\gamma^{1.02}}{1+\left(\gamma / \gamma_{\mathrm{c}}\right)^{2.02}}$

$\mathrm{cm}^{-3}$ (unit Lorentz factor) ${ }^{-1}$

with

$n_{\mathrm{e}, 0}=1.3 \frac{n_{\mathrm{i}}^{*} \Gamma_{2}}{n_{\mathrm{b}, 10}^{*} d_{13}^{*}} \quad \mathrm{~cm}^{-3}$ (unit Lorentz factor) $^{-2.02}$

which is suitable to calculate the electrostatic bremsstrahlung and synchrotron radiation intensities. The total relativistic electron density is

$$
\begin{aligned}
n_{\mathrm{e}, \text { tot }} & =\int_{1}^{\infty} \mathrm{d} \gamma n_{\mathrm{e}}(\gamma)=n_{\mathrm{e}, 0} \int_{1}^{\Gamma} \mathrm{d} \gamma \frac{\gamma^{1.02}}{1+\left(\gamma / \gamma_{\mathrm{c}}\right)^{2.02}} \\
& \simeq n_{\mathrm{e}, 0} \gamma_{\mathrm{c}}^{2.02}\left[\ln \frac{\Gamma}{\gamma_{\mathrm{c}}}+\frac{1}{2.02}\right] \mathrm{cm}^{-3} .
\end{aligned}
$$

\section{Electrostatic bremsstrahlung and synchrotron radiation from relativistic pick-up electrons}

\subsection{Monochromatic approximations for electrostatic bremsstrahlung and synchrotron radiation}

For relativistic electron energies the monochromatic approximation for electrostatic bremsstrahlung (57) reads

$$
\tilde{p}_{\mathrm{eb}}(v, \gamma) \simeq \frac{128}{27} \sigma_{\mathrm{T}} c U_{\mathrm{L}} \gamma^{2} \delta\left(v-\frac{7}{5} \nu_{\mathrm{p}} \gamma^{2}\right) \quad \mathrm{erg} \mathrm{s}^{-1} \mathrm{~Hz}^{-1}
$$

The corresponding monochromatic approximation for synchrotron radiation is derived in Appendix B and reads

$$
\tilde{p}_{\text {sy }}(\nu, \gamma) \simeq 2 \sigma_{\mathrm{T}} c U_{\mathrm{B}} \gamma^{2} \exp \left(-\gamma_{\mathrm{R}} / \gamma\right) \delta\left(v-v_{\mathrm{p}} \gamma\left[1+\frac{\gamma}{\gamma_{\mathrm{R}}}\right]\right)
$$

$\operatorname{erg~} \mathrm{s}^{-1} \mathrm{~Hz}^{-1}$

with the Razin-Tsytovich Lorentz factor (78).

\subsection{Spontaneous electrostatic bremsstrahlung}

With Eqs. (89) and (92) the spontaneous electrostatic bremsstrahlung emission coefficient of relativistic electrons is

$$
\begin{aligned}
j_{\mathrm{eb}}(v) & =\frac{1}{4 \pi} \int_{1}^{\infty} \mathrm{d} \gamma n_{\mathrm{e}}(\gamma) \tilde{p}_{\mathrm{eb}}(v, \gamma) \\
& =\frac{32}{27 \pi} \sigma_{\mathrm{T}} c U_{\mathrm{L}} \int_{1}^{\infty} \mathrm{d} \gamma n_{\mathrm{e}}(\gamma) \gamma^{2} \delta\left(\nu-\frac{7}{5} v_{\mathrm{p}} \gamma^{2}\right) \\
& =\frac{80}{189 \pi} \frac{\sigma_{\mathrm{T}} c U_{\mathrm{L}}}{v_{\mathrm{p}}} \int_{1}^{\infty} \mathrm{d} \gamma n_{\mathrm{e}}(\gamma) \gamma \delta\left(\gamma-\gamma_{\mathrm{b}}\right) \\
& =\frac{80}{189 \pi} \frac{\sigma_{\mathrm{T}} c U_{\mathrm{L}}}{v_{\mathrm{p}}} \gamma_{\mathrm{b}} n_{\mathrm{e}}\left(\gamma_{\mathrm{b}}\right) \mathrm{erg} \mathrm{s}^{-1} \mathrm{~Hz}^{-1} \mathrm{ster}^{-1} \mathrm{~cm}^{-3}
\end{aligned}
$$

for

$\gamma_{\mathrm{b}}=\sqrt{\frac{5 v}{7 v_{\mathrm{p}}}} \geq 1$
We obtain

$$
\begin{aligned}
j_{\mathrm{eb}}(v)= & \frac{80}{189 \pi} \frac{\sigma_{\mathrm{T}} c U_{\mathrm{L}} n_{\mathrm{e}, 0}}{v_{\mathrm{p}}} \frac{\left[\frac{5 v}{7 v_{\mathrm{p}}}\right]^{1.01}}{1+\left[\frac{5 v}{7 v_{\mathrm{p}} \gamma_{\mathrm{c}}^{2}}\right]^{1.01}} \\
& \times H\left[v-\frac{7}{5} v_{\mathrm{p}}\right] H\left[\frac{7}{5} v_{\mathrm{p}} \Gamma^{2}-v\right] \\
\propto & \begin{cases}v^{1.01} & \text { for } \frac{7}{5} v_{\mathrm{p}} \leq v \leq \frac{7}{5} v_{\mathrm{p}} \gamma_{\mathrm{c}}^{2} \\
v^{0} & \text { for } \frac{7}{5} v_{\mathrm{p}} \gamma_{\mathrm{c}}^{2}<v \leq \frac{7}{5} v_{\mathrm{p}} \Gamma^{2} . \\
0 & \text { otherwise }\end{cases}
\end{aligned}
$$

\subsection{Spontaneous synchrotron radiation}

With Eqs. (89) and (93) the spontaneous synchrotron emission coefficient of relativistic electrons is

$$
\begin{aligned}
j_{\mathrm{sy}}(v)= & \frac{1}{4 \pi} \int_{1}^{\infty} \mathrm{d} \gamma n_{\mathrm{e}}(\gamma) \tilde{p}_{\mathrm{sy}}(v, \gamma) \\
= & \frac{\sigma_{\mathrm{T}} c U_{\mathrm{B}}}{2 \pi} \int_{1}^{\infty} \mathrm{d} \gamma n_{\mathrm{e}}(\gamma) \gamma^{2} \exp \left(-\frac{\gamma_{\mathrm{R}}}{\gamma}\right) \\
& \times \delta\left(v-v_{\mathrm{p}} \gamma\left[1+\frac{\gamma}{\gamma_{\mathrm{R}}}\right]\right) \\
= & \frac{1}{4 \pi} \frac{\sigma_{\mathrm{T}} c U_{\mathrm{B}}}{v_{\mathrm{p}}} \int_{1}^{\infty} \mathrm{d} \gamma \frac{n_{\mathrm{e}}(\gamma) \gamma^{2}}{1+\frac{2 \gamma}{\gamma_{\mathrm{R}}}} \exp \left(-\frac{\gamma_{\mathrm{R}}}{\gamma}\right) \delta\left(\gamma-\gamma_{\mathrm{s}}\right) \\
= & \frac{1}{4 \pi} \frac{\sigma_{\mathrm{T}} c U_{\mathrm{B}}}{v_{\mathrm{p}}} \frac{\gamma_{\mathrm{s}}^{2} n_{\mathrm{e}}\left(\gamma_{\mathrm{s}}\right)}{1+\frac{2 \gamma_{\mathrm{s}}}{\gamma_{\mathrm{R}}}} \exp \left(-\frac{\gamma_{\mathrm{R}}}{\gamma_{\mathrm{s}}}\right) \\
& \operatorname{erg~s}^{-1} \mathrm{~Hz}^{-1} \operatorname{ster}^{-1} \mathrm{~cm}^{-3}
\end{aligned}
$$

for

$1 \leq \gamma_{\mathrm{s}}=\frac{v_{\mathrm{p}}}{3 v_{0}}\left[\sqrt{1+\frac{6 v v_{0}}{v_{\mathrm{p}}^{2}}}-1\right] \simeq \begin{cases}\frac{v}{v_{\mathrm{p}}} & \text { for } v \leq v_{\mathrm{R}} \\ \sqrt{\frac{2 v}{3 v_{0}}} & \text { for } v>v_{\mathrm{R}}\end{cases}$

where

$v_{\mathrm{R}} \equiv \frac{v_{\mathrm{p}}^{2}}{6 v_{0}}=10^{9} \frac{n_{\mathrm{b}, 8}}{\left(B_{0} / \text { Gauss }\right)} \mathrm{Hz}$

is the Razin frequency in a pair plasma. We obtain

$j_{\mathrm{sy}}(v)=\frac{\sigma_{\mathrm{T}} c U_{\mathrm{B}} \gamma_{\mathrm{s}}^{2} n_{\mathrm{e}}\left(\gamma_{\mathrm{s}}\right)}{4 \pi v_{\mathrm{p}} \sqrt{1+\frac{v}{v_{\mathrm{R}}}}} \exp \left(-\frac{2}{\sqrt{1+\frac{v}{\nu_{\mathrm{R}}}}-1}\right)$.

Equation (100) shows that for frequencies $v<v_{\mathrm{R}}$ the synchrotron emission is exponentially reduced, whereas at larger frequencies $v>v_{\mathrm{R}}$ we obtain

$$
\begin{aligned}
j_{\mathrm{sy}}\left(v>v_{\mathrm{R}}\right) \simeq & \frac{\sigma_{\mathrm{T}} c U_{\mathrm{B}} n_{\mathrm{e}, 0}}{4 \pi v_{\mathrm{s}}} \frac{\left[\frac{v}{v_{\mathrm{s}}}\right]^{1.01}}{1+\left[\frac{v}{v_{\mathrm{s}} \gamma_{\mathrm{c}}^{2}}\right]^{1.01}} H\left[v-v_{\mathrm{R}}\right] H\left[v_{\mathrm{s}} \Gamma^{2}-v\right] \\
& \propto \begin{cases}v^{1.01} & \text { for } v_{\mathrm{s}} \leq v \leq v_{\mathrm{s}} \gamma_{\mathrm{c}}^{2} \\
v^{0} & \text { for } v_{\mathrm{s}} \gamma_{\mathrm{c}}^{2}<v \leq v_{\mathrm{S}} \Gamma^{2} \\
0 & \text { otherwise }\end{cases}
\end{aligned}
$$

where

$v_{\mathrm{s}}=\frac{3}{2} v_{0}=4.2 \times 10^{6}\left(B_{0} /\right.$ Gauss $) \quad \mathrm{Hz}$. 


\subsection{Electrostatic bremsstrahlung absorption coefficient}

With Eqs. (89) and (92) the electrostatic bremsstrahlung absorption coefficient of relativistic electrons is

$$
\begin{aligned}
& \alpha_{\mathrm{eb}}(v) \simeq-\frac{c^{2}}{2 m_{\mathrm{e}} c^{2}\left(v^{2}-v_{\mathrm{p}}^{2}\right)} \int_{1}^{\infty} \mathrm{d} \gamma \gamma^{2} \tilde{p}_{\mathrm{eb}}(v, \gamma) \frac{\mathrm{d}}{\mathrm{d} \gamma}\left[\frac{n_{\mathrm{e}}(\gamma)}{\gamma^{2}}\right] \\
&=-\frac{64}{27} \frac{\sigma_{\mathrm{T}} c^{3} U_{\mathrm{L}}}{m_{\mathrm{e}} c^{2}\left(v^{2}-v_{\mathrm{p}}^{2}\right)} \\
& \times \int_{1}^{\infty} \mathrm{d} \gamma \gamma^{4} \delta\left(v-\frac{7}{5} v_{\mathrm{p}} \gamma^{2}\right) \frac{\mathrm{d}}{\mathrm{d} \gamma}\left[\frac{n_{\mathrm{e}}(\gamma)}{\gamma^{2}}\right] \\
&= \frac{160}{189} \frac{\sigma_{\mathrm{T}} c^{3} U_{\mathrm{L}} n_{\mathrm{e}, 0}}{m_{\mathrm{e}} c^{2} v_{\mathrm{p}}\left(v^{2}-v_{\mathrm{p}}^{2}\right) \gamma_{\mathrm{c}}^{1.98}} \int_{1}^{\infty} \mathrm{d} \gamma \gamma^{3} \\
&\left.\left.\times \delta\left(\gamma-\gamma_{\mathrm{b}}\right) \frac{\left[3\left(\frac{\gamma}{\gamma_{\mathrm{c}}}\right)^{0.04}+0.98\left(\frac{\gamma}{\gamma_{\mathrm{c}}}\right)^{-1.98}\right]}{\gamma^{2}}\right]^{2.02}\right]^{2} \\
&= \frac{160}{189} \frac{\sigma_{\mathrm{T}} c^{3} U_{\mathrm{L}} n_{\mathrm{e}, 0}}{m_{\mathrm{e}} c^{2} v_{\mathrm{p}}\left(v^{2}-v_{\mathrm{p}}^{2}\right) \gamma_{\mathrm{c}}^{1.98} \gamma_{\mathrm{b}}^{3}} \\
& \times \frac{\left[3\left(\frac{\gamma_{\mathrm{b}}}{\gamma_{\mathrm{c}}}\right)^{0.04}+0.98\left(\frac{\gamma_{\mathrm{b}}}{\gamma_{\mathrm{c}}}\right)^{-1.98}\right]}{\mathrm{cm}^{-1}} \\
& {\left[1+\left(\frac{\gamma_{\mathrm{b}}}{\gamma_{\mathrm{c}}}\right)^{2.02}\right]^{2} }
\end{aligned}
$$

for $\gamma_{\mathrm{b}} \geq 1$. We obtain

$$
\begin{aligned}
\alpha_{\mathrm{eb}}(v)= & \frac{160}{189} \frac{\sigma_{\mathrm{T}} c^{3} U_{\mathrm{L}} n_{\mathrm{e}, 0}}{m_{\mathrm{e}} c^{2} v_{\mathrm{p}}\left(v^{2}-v_{\mathrm{p}}^{2}\right) \gamma_{\mathrm{c}}^{1.98}}\left[\frac{5 v}{7 v_{\mathrm{p}}}\right]^{3 / 2} \\
& \times \frac{\left[3\left(\frac{5 v}{7 v_{\mathrm{p}} \gamma_{\mathrm{c}}^{2}}\right)^{0.02}+0.98\left(\frac{5 v}{7 v_{\mathrm{p}} \gamma_{\mathrm{c}}^{2}}\right)^{-0.99}\right]}{\left[1+\left(\frac{5 v}{7 v_{\mathrm{p}} \gamma_{\mathrm{c}}^{2}}\right)^{1.01}\right]^{2}} .
\end{aligned}
$$

The optical depth for the uniform outflow region of length $L$ then is

$$
\begin{aligned}
\tau_{\mathrm{eb}}(v)= & L \alpha_{\mathrm{eb}}(v)=\frac{160}{189} \frac{\sigma_{\mathrm{T}} c^{3} L U_{\mathrm{L}} n_{\mathrm{e}, 0}}{m_{\mathrm{e}} c^{2} v_{\mathrm{p}}\left(v^{2}-v_{\mathrm{p}}^{2}\right) \gamma_{\mathrm{c}}^{1.98}}\left[\frac{5 v}{7 v_{\mathrm{p}}}\right]^{3 / 2} \\
& \times \frac{\left[3\left(\frac{5 v}{7 v_{\mathrm{p}} \gamma_{\mathrm{c}}^{2}}\right)^{0.02}+0.98\left(\frac{5 v}{7 v_{\mathrm{p}} \gamma_{\mathrm{c}}^{2}}\right)^{-0.99}\right]}{\left[1+\left(\frac{5 v}{7 v_{\mathrm{p}} \gamma_{\mathrm{c}}^{2}}\right)^{1.01}\right]^{2}} \\
= & 5 \times 10^{-9} d_{13}^{*}\left(n_{\mathrm{i}}^{*}\right)^{2.99} \Gamma_{2}^{8.47}\left(n_{\mathrm{b}, 10}^{*}\right)^{-3.49} \frac{\left[\frac{v}{v_{\mathrm{p}}}\right]^{3 / 2}}{\left[\left(\frac{v}{v_{\mathrm{p}}}\right)^{2}-1\right]} \\
& \times \frac{\left[3\left(\frac{5 v}{7 v_{\mathrm{p}} \gamma_{\mathrm{c}}^{2}}\right)^{0.02}+0.98\left(\frac{5 v}{7 v_{\mathrm{p}} \gamma_{\mathrm{c}}^{2}}\right)^{-0.99}\right]}{\left[1+\left(\frac{5 v}{7 v_{\mathrm{p}} \gamma_{\mathrm{c}}^{2}}\right)^{1.01}\right]^{2}}
\end{aligned}
$$

$$
\propto \begin{cases}\frac{v^{0.51}}{v^{2}-v_{\mathrm{p}}^{2}} & \text { for } \frac{7}{5} v_{\mathrm{p}} \leq v \leq \frac{7}{5} v_{\mathrm{p}} \gamma_{\mathrm{c}}^{2} \\ \frac{v^{-0.5}}{v^{2}-v_{\mathrm{p}}^{2}} & \text { for } \frac{7}{5} v_{\mathrm{p}} \gamma_{\mathrm{c}}^{2}<v \leq \frac{7}{5} v_{\mathrm{p}} \Gamma^{2} . \\ 0 & \text { otherwise }\end{cases}
$$

This optical depth is smaller than the optical depth (69) from bulk electrostatic bremsstrahlung at frequencies less than $v_{\mathrm{b}}=$ $15.2 v_{\mathrm{p}}$ for standard jet outflow parameters. Above this frequency the optical depth (105) itself is much smaller than unity. Therefore the optical depth from relativistic electrostatic bremsstrahlung can be neglected compared to the optical depth from bulk electrostatic bremsstrahlung. As a consequence, relativistic electrostatic bremsstrahlung is optically thick at at all frequencies $v_{\mathrm{p}} \leq v \leq v_{\mathrm{c}}$, where $v_{\mathrm{c}}$ is given by Eq. (70).

\subsection{Synchrotron absorption coefficient}

In similar manner we obtain for the synchrotron absorption coefficient of relativistic electrons

$$
\begin{aligned}
\alpha_{\mathrm{sy}}(v)= & -\frac{\sigma_{\mathrm{T}} c^{3} U_{\mathrm{B}}}{m_{\mathrm{e}} c^{2}\left(v^{2}-v_{\mathrm{p}}^{2}\right) v_{\mathrm{p}}} \\
& \times \int_{1}^{\infty} \mathrm{d} \gamma \frac{\gamma^{4}}{1+\frac{2 \gamma}{\gamma_{\mathrm{R}}}} \exp \left(-\frac{\gamma_{\mathrm{R}}}{\gamma}\right) \delta\left(\gamma-\gamma_{\mathrm{s}}\right) \frac{\mathrm{d}}{\mathrm{d} \gamma}\left[\frac{n_{\mathrm{e}}(\gamma)}{\gamma^{2}}\right] \\
= & \frac{\sigma_{\mathrm{T}} c^{3} U_{\mathrm{B}} n_{\mathrm{e}, 0}}{v_{\mathrm{p}} m_{\mathrm{e}} c^{2}\left(v^{2}-v_{\mathrm{p}}^{2}\right) \gamma_{\mathrm{c}}^{1.98}} \frac{\gamma_{\mathrm{s}}^{4}}{1+\frac{2 \gamma_{\mathrm{s}}}{\gamma_{\mathrm{R}}}} \exp \left(-\frac{2}{\sqrt{1+\frac{v}{\gamma_{\mathrm{R}}}}-1}\right) \\
& \times \frac{\left[3\left(\frac{\gamma_{\mathrm{s}}}{\gamma_{\mathrm{c}}}\right)^{0.04}+0.98\left(\frac{\gamma_{\mathrm{s}}}{\gamma_{\mathrm{c}}}\right)^{-1.98}\right]}{\left[1+\left(\frac{\gamma_{\mathrm{s}}}{\gamma_{\mathrm{c}}}\right)^{2.02}\right]^{2}} .
\end{aligned}
$$

Again, Eq. (106) shows that for frequencies $v<v_{\mathrm{R}}$ the synchrotron absorption coefficient emission is exponentially reduced, whereas at larger frequencies $v>v_{\mathrm{R}}$ we obtain

$$
\begin{aligned}
\alpha_{\mathrm{sy}}\left(v>v_{\mathrm{R}}\right) \simeq & \frac{\sigma_{\mathrm{T}} c^{3} U_{\mathrm{B}} n_{\mathrm{e}, 0}}{2 v_{\mathrm{s}} m_{\mathrm{e}} c^{2}\left(v^{2}-v_{\mathrm{p}}^{2}\right) \gamma_{\mathrm{c}}^{1.98}}\left[\frac{v}{v_{\mathrm{s}}}\right]^{\frac{3}{2}} \\
& \times \frac{\left[3\left(\frac{v}{v_{\mathrm{s}} \gamma_{\mathrm{c}}^{2}}\right)^{0.02}+0.98\left(\frac{v}{v_{\mathrm{s}} \gamma_{\mathrm{c}}^{2}}\right)^{-0.99}\right]}{\left[1+\left(\frac{v}{v_{\mathrm{s}} \gamma_{\mathrm{c}}^{2}}\right)^{1.01}\right]^{2}}
\end{aligned}
$$

so that the corresponding optical depth is

$$
\begin{aligned}
\tau_{\mathrm{sy}}\left(v>v_{\mathrm{R}}\right)= & L \alpha_{\mathrm{sy}}\left(v>v_{\mathrm{R}}\right)=\frac{\sigma_{\mathrm{T}} c^{3} L U_{\mathrm{B}} n_{\mathrm{e}, 0}}{2 m_{\mathrm{e}} c^{2} v_{\mathrm{s}}\left(v^{2}-v_{\mathrm{p}}^{2}\right) \gamma_{\mathrm{c}}^{1.98}} \\
& \times\left[\frac{v}{v_{\mathrm{s}}}\right]^{3 / 2} \frac{\left[3\left(\frac{v}{v_{\mathrm{s}} \gamma_{\mathrm{c}}^{2}}\right)^{0.02}+0.98\left(\frac{v}{v_{\mathrm{s}} \gamma_{\mathrm{c}}^{2}}\right)^{-0.99}\right]}{\left[1+\left(\frac{v}{v_{\mathrm{s}} \gamma_{\mathrm{c}}^{2}}\right)^{1.01}\right]^{2}} \\
\propto & \begin{cases}\frac{v^{0.51}}{v^{2}-v_{\mathrm{p}}^{2}} & \text { for } v_{\mathrm{s}} \leq v \leq v_{\mathrm{s}} \gamma_{\mathrm{c}}^{2} \\
\frac{v^{-0.5}}{v^{2}-v_{\mathrm{p}}^{2}} & \text { for } v_{\mathrm{s}} \gamma_{\mathrm{c}}^{2}<v \leq v_{\mathrm{s}} \Gamma^{2} \\
0 & \text { otherwise }\end{cases}
\end{aligned}
$$




\subsection{Frequency domains}

From the respective emission and absorption coefficients we note that electrostatic bremsstrahlung and synchrotron radiation from relativistic electrons operate at different frequency regimes.

The synchrotron emission of relativistic electrons occurs at co-moving photon frequencies between

$v_{\mathrm{R}} \leq v \leq v_{\mathrm{S}} \Gamma^{2}$

which for standard inner jet outflow parameters $\left(B_{0}=1\right.$ Gauss, $n_{\mathrm{b}, 8}=1, \Gamma=100 \Gamma_{2}$ ) corresponds to

$1 \mathrm{GHz} \leq v \leq 42 \mathrm{GHz}$.

Electrostatic bremsstrahlung from relativistic electrons occurs at co-moving photon frequencies between

$1.4 v_{\mathrm{p}} \leq v \leq 1.4 v_{\mathrm{p}} \Gamma^{2}$

which for standard jet outflow parameters corresponds to

$130 \mathrm{MHz} \leq v \leq 1300 \mathrm{GHz}$.

For standard inner jet outflow parameters, the emission and absorption coefficients and the optical depth of synchrotron radiation are orders of magnitude smaller than the corresponding emission and absorption coefficients and the optical depth of electrostatic bremsstrahlung by relativistic electrons. As demonstrated, the electrostatic bremsstrahlung optical depth by relativistic electrons in turn is smaller than unity at frequencies larger than $v_{\mathrm{b}}=15.2 v_{\mathrm{p}}$ and below this frequency smaller than the bulk electrostatic bremsstrahlung optical depth. Consequently, relativistic synchrotron radiation and relativistic electrostatic bremsstrahlung are optically thin for frequencies above $v_{\mathrm{c}}$, and optically thick below.

\subsection{Intensity of electrostatic bremsstrahlung and synchrotron radiation from relativistic electrons}

Electrostatic bremsstrahlung is optically thin at all frequencies larger $v_{\mathrm{c}}$, i.e.

$$
\begin{aligned}
& I_{\mathrm{eb}}\left(v \geq v_{\mathrm{c}}\right)=j_{\mathrm{eb}}(v) L=\frac{80}{189 \pi} \frac{\sigma_{\mathrm{T}} c L U_{\mathrm{L}} n_{\mathrm{e}, 0}}{v_{\mathrm{p}}}
\end{aligned}
$$

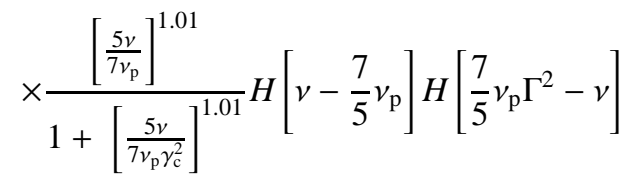

$$
\begin{aligned}
& \propto L U_{\mathrm{L}} n_{\mathrm{e}, 0} \begin{cases}v^{1.01} & \text { for } \frac{7}{5} v_{\mathrm{p}} \leq v \leq \frac{7}{5} v_{\mathrm{p}} \gamma_{\mathrm{c}}^{2} \\
v^{0} & \text { for } \frac{7}{5} v_{\mathrm{p}} \gamma_{\mathrm{c}}^{2}<v \leq \frac{7}{5} v_{\mathrm{p}} \Gamma^{2} . \\
0 & \text { otherwise }\end{cases}
\end{aligned}
$$

Using Eq. (68) in the optically thick regime, the relativistic electrostatic bremsstrahlung intensity is given by

$$
\begin{aligned}
& I_{\mathrm{eb}}\left(1.4 v_{\mathrm{p}} \leq v<v_{\mathrm{c}}\right) \simeq \frac{j_{\mathrm{eb}}(v)}{n_{\mathrm{r}}^{2} \alpha(v)} \\
& =\frac{80}{189}\left(\frac{5}{7}\right)^{1.01} \sqrt{\frac{3}{2 \pi}} m_{\mathrm{e}} \Theta^{5 / 2} v_{\mathrm{p}}^{2} \frac{n_{\mathrm{e}, 0}}{n_{\mathrm{b}}}\left[\frac{v}{v_{\mathrm{p}}}-1\right]^{-1 / 2}
\end{aligned}
$$

$$
\times\left[\frac{v}{v_{\mathrm{p}}}+1\right]^{-1} \exp \left(\frac{3\left(v-v_{\mathrm{p}}\right)}{8 \Theta v_{\mathrm{p}}}\right) \frac{\left[\frac{v}{v_{\mathrm{p}}}\right]^{3.01}}{1+\left[\frac{5 v}{7 v_{\mathrm{p}} \gamma_{\mathrm{c}}^{2}}\right]^{1.01}} .
$$

Synchrotron radiation in the optically thin region is

$$
\begin{aligned}
I_{\mathrm{sy}}\left(v \geq v_{\mathrm{c}}\right) & =j_{\mathrm{sy}}\left(v>v_{\mathrm{c}}\right) L \\
& =\frac{\sigma_{\mathrm{T}} c L U_{\mathrm{B}}}{4 \pi v_{\mathrm{p}} \sqrt{1+\frac{v}{v_{\mathrm{R}}}}} \gamma_{\mathrm{s}}^{2} n_{\mathrm{e}}\left(\gamma_{\mathrm{s}}\right) \exp \left(-\frac{2}{\sqrt{1+\frac{v}{v_{\mathrm{R}}}}-1}\right)
\end{aligned}
$$

whereas in the optically thick region

$I_{\mathrm{sy}}\left(v_{\mathrm{p}} \leq v<v_{\mathrm{c}}\right) \simeq \frac{j_{\mathrm{sy}}(v)}{n_{\mathrm{r}}^{2} \alpha(v)}$

$$
\begin{aligned}
= & \sqrt{\frac{3}{32 \pi}} \frac{U_{\mathrm{B}}}{U_{\mathrm{L}} n_{\mathrm{b}}} \frac{\gamma_{\mathrm{s}}^{2} n_{\mathrm{e}}\left(\gamma_{\mathrm{s}}\right)}{\sqrt{1+\frac{v}{v_{\mathrm{R}}}}} \exp \left(-\frac{2}{\sqrt{1+\frac{v}{v_{\mathrm{R}}}}-1}\right) \\
& \times\left[\frac{v}{v_{\mathrm{p}}}-1\right]^{-1 / 2}\left[\frac{v}{v_{\mathrm{p}}}+1\right]^{-1} \exp \left(\frac{3\left(v-v_{\mathrm{p}}\right)}{8 \Theta v_{\mathrm{p}}}\right) .
\end{aligned}
$$

Both intensity distributions are shown in Fig. 1.

\section{Doppler boosted electrostatic bremsstrahlung and synchrotron radiation}

After having determined the intensities of bulk pair electrostatic bremsstrahlung (Sect. 5) and the electrostatic bremsstrahlung and synchrotron radiation of the relativistic pick-up electrons (Sect. 7) in the co-moving frame, we now finally transform to the observer's frame using the invariance (Rybicki \& Lightman 1979; Dermer \& Schlickeiser 2002) of

$$
\frac{I(v)}{v^{3}}=\frac{I^{*}\left(v^{*}\right)}{\left(v^{*}\right)^{3}}
$$

and

$v^{*}=D v$

where the Doppler factor

$D=\left[\Gamma\left(1-B \mu^{*}\right)\right]^{-1}$

depends on the cosine of the observer's viewing angle $\mu^{*}=$ $\cos \theta^{*}$ with respect to the jet propagation direction $(V=B c)$. Our discussion of the optical depth effects (see Eq. (70)) has shown that all three emission processes are optically thin at frequencies above $v_{\mathrm{c}}$. An observer looking at an optically thin source of comoving volume $V_{\mathrm{b}}$ at redshift $z$ measures a flux density $S^{*}\left(v^{*}\right)$ given by (Dermer et al. 1997)

$S^{*}\left(v^{*}\right)=\frac{D^{3}(1+z) V_{\mathrm{b}}}{d_{\mathrm{L}}^{2}} j\left(\frac{v^{*}(1+z)}{D}\right)$

$\operatorname{erg~} \mathrm{cm}^{-2} \mathrm{~s}^{-1} \mathrm{~Hz}^{-1}$

where $d_{\mathrm{L}}=2 c\left[z+1-(z+1)^{1 / 2}\right] / H_{0}$ is the luminosity distance for a critical density universe with zero cosmological constant. 
This transformation will be used in the following to determine the luminosity photon spectra $v^{*} S^{*}\left(v^{*}\right)$.

According to Eqs. (66) and (71) the bulk pair electrostatic bremsstrahlung in the observer's frame is

$v^{*} S^{*}\left(v^{*}>v_{\mathrm{c}}^{*}\right)=\frac{D^{4}(1+z) V_{\mathrm{b}}}{6^{1 / 2} \pi^{3 / 2} d_{\mathrm{L}}^{2}} \frac{\sigma_{\mathrm{T}} c U_{\mathrm{L}} n_{\mathrm{b}}}{\Theta^{3 / 2}}$

$H\left[v^{*}-v_{\mathrm{p}}^{*}\right]\left[\frac{v^{*}}{v_{\mathrm{p}}^{*}}-1\right]^{1 / 2} \frac{v^{*}}{v_{\mathrm{p}}^{*}}\left(\frac{v^{*}}{v_{\mathrm{p}}^{*}}+1\right) \exp \left(-\frac{3\left(v^{*}-v_{\mathrm{p}}^{*}\right)}{8 \Theta v_{\mathrm{p}}^{*}}\right)$

at optically thin frequencies range, whereas in the optically thick regime

$v^{*} S^{*}\left(v_{\mathrm{p}}^{*} \leq v^{*} \leq v_{\mathrm{c}}^{*}\right) \propto\left(v^{*}\right)^{3}$.

Likewise, from Eqs. (96) and (101) we derive for the observed electrostatic bremsstrahlung and synchrotron radition from the relativistic electrons

$$
\begin{aligned}
v^{*} S_{\mathrm{eb}}^{*}\left(v^{*} \geq v_{\mathrm{c}}^{*}\right) & =\frac{80 D^{4}(1+z) V_{\mathrm{b}}}{189 \pi d_{\mathrm{L}}^{2}} \sigma_{\mathrm{T}} c U_{\mathrm{L}} n_{\mathrm{e}, 0} \frac{v^{*}}{v_{\mathrm{p}}^{*}} \\
& \times \frac{\left[\frac{5 v^{*}}{7 v_{\mathrm{p}}^{*}}\right]^{1.01}}{1+\left[\frac{5 v^{*}}{7 v_{\mathrm{p}}^{*} \gamma_{\mathrm{c}}^{2}}\right]^{1.01} H\left[v^{*}-\frac{7}{5} v_{\mathrm{p}}^{*}\right] H\left[\frac{7}{5} v_{\mathrm{p}}^{*} \Gamma^{2}-v^{*}\right]} \\
& \propto \frac{D^{4}(1+z) V_{\mathrm{b}} U_{\mathrm{L}} n_{\mathrm{e}, 0}}{d_{\mathrm{L}}^{2}} \\
& \times \begin{cases}\left(v^{*}\right)^{2.01} & \text { for } \frac{7}{5} v_{\mathrm{p}}^{*} \leq v^{*} \leq \frac{7}{5} v_{\mathrm{p}}^{*} \gamma_{\mathrm{c}}^{2} \\
v^{*} & \text { for } \frac{7}{5} v_{\mathrm{p}}^{*} \gamma_{\mathrm{c}}^{2}<v^{*} \leq \frac{7}{5} v_{\mathrm{p}}^{*} \Gamma^{2} \\
0 & \text { otherwise }\end{cases}
\end{aligned}
$$

and

$$
\begin{aligned}
v^{*} S_{\mathrm{sy}}^{*}\left(v^{*} \geq v_{\mathrm{R}}^{*}\right)= & \frac{D^{4}(1+z) V_{\mathrm{b}}}{4 \pi d_{\mathrm{L}}^{2}} \sigma_{\mathrm{T}} c U_{\mathrm{B}} n_{\mathrm{e}, 0} \\
& \times \exp \left(-\frac{4 v_{\mathrm{R}}^{*}}{v^{*}}\right) \frac{\left[\frac{v^{*}}{v_{\mathrm{s}}^{*}}\right]^{2.01}}{1+\left[\frac{v^{*}}{v_{\mathrm{s}}^{*} \gamma_{\mathrm{c}}^{2}}\right]^{1.01}} \\
& \times H\left[v^{*}-v_{\mathrm{s}}^{*}\right] H\left[v_{\mathrm{s}}^{*} \Gamma^{2}-v^{*}\right] \\
\propto & \frac{D^{4}(1+z) V_{\mathrm{b}} U_{\mathrm{B}} n_{\mathrm{e}, 0}}{d_{\mathrm{L}}^{2}} \\
& \times \begin{cases}\left(v^{*}\right)^{2.01} & \text { for } v_{\mathrm{R}}^{*} \leq v^{*} \leq v_{\mathrm{s}} * \gamma_{\mathrm{c}}^{2} \\
v^{*} & \text { for } v_{\mathrm{s}}^{*} \gamma_{\mathrm{c}}^{2}<v^{*} \leq v_{\mathrm{s}}^{*} \Gamma^{2} . \\
0 & \text { otherwise }\end{cases}
\end{aligned}
$$

The respective $v^{*} S^{*}$-distributions for standard inner jet parameters in the observer's frame are shown in Fig. 1. The luminosity spectra are normalised in units of

$$
\begin{aligned}
& L_{0}=D^{4}(1+z) V_{\mathrm{b}} \sigma_{\mathrm{T}} c U_{\mathrm{L}} n_{\mathrm{b}} / d_{\mathrm{L}}^{2} \\
& =7.5 \times 10^{-17} \frac{D^{4} R_{15}^{2} d_{13}^{*} n_{\mathrm{i}}^{*} n_{\mathrm{b}, 10}^{*} \Gamma_{2}^{2} h_{75}^{2}}{[\sqrt{1+z}-1]^{2}} \mathrm{erg} \mathrm{cm}^{-2} \mathrm{~s}^{-1}
\end{aligned}
$$

where we used a Hubble constant of $H_{0}=75 h_{75} \mathrm{~km} \mathrm{~s}^{-1} \mathrm{Mpc}^{-1}$.

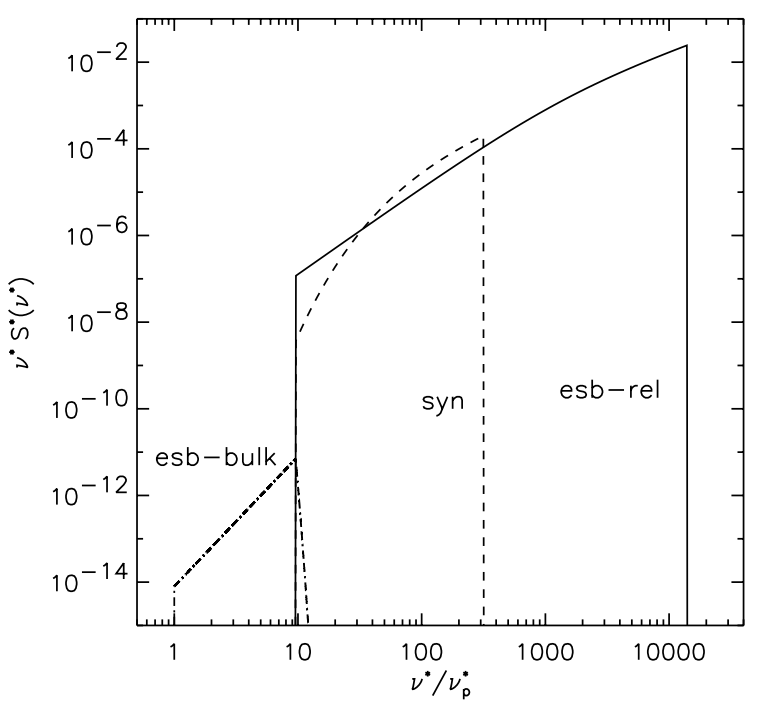

Fig. 1. Doppler boosted luminosity spectra $v^{*} S^{*}\left(v^{*}\right)$ in units of $L_{0}=$ $D^{4}(1+z) V_{\mathrm{b}} \sigma_{\mathrm{T}} c U_{\mathrm{L}} n_{\mathrm{b}} / d_{\mathrm{L}}^{2}$ as function of the normalised frequency $v^{*} / v_{\mathrm{p}}^{*}$ for bulk electrostatic bremsstrahlung, synchrotron radiation and relativistic electrostatic bremsstrahlung.

Bulk electrostatic bremsstrahlung appears as narrow-band (width $\left.\Delta v^{*} \simeq\left(v_{\mathrm{c}}^{*}-v_{\mathrm{p}}^{*}\right) \simeq 10 v_{\mathrm{p}}^{*}\right)$ feature just above the Doppler boosted plasma frequency $v_{\mathrm{p}}^{*}=0.13 \mathrm{D} \leq 26 \Gamma_{2} \mathrm{GHz}$, where the upper limits hold for emission in the forward direction.

Relativistic electrostatic bremsstrahlung has a wide frequency distribution peaking at the highest frequency of

$v_{\text {max }}^{*}=1.4 v_{\mathrm{p}}^{*} \Gamma^{2}=1.4 v_{\mathrm{p}} D \Gamma^{2}$

which becomes

$v_{\text {max }}^{*}=2.8 v_{\mathrm{p}} \Gamma^{3}=2.6 \times 10^{14} n_{\mathrm{b}, 10}^{*} \Gamma_{2}^{2} \mathrm{~Hz}$

in the forward direction $\left(\mu^{*}=1\right)$, which lies well in the optical band for standard inner jet outflow parameters.

Synchrotron radiation is also broadband. At low frequencies the Razin suppression is clearly visible, and the maximum frequency occurs at

$v_{\text {max }, \mathrm{s}}^{*}=v_{\mathrm{s}}^{*} \Gamma^{2}=4.2 \times 10^{6} B_{0} D \Gamma^{2} \leq 6.7 \times 10^{13} B_{0} \Gamma_{2}^{3}$

Relativistic electrostatic bremsstrahlung and synchrotron radiation have much higher peak luminosities than bulk electrostatic bremsstarhlung with $v^{*} S_{\mathrm{eb}}^{*}\left(v^{*}\right) \simeq 10^{-2} L_{0}$ and $v^{*} S_{\mathrm{sy}}^{*}\left(v^{*}\right) \simeq$ $10^{-4} L_{0}$. For standard inner jet outflow parameters at a redshift of $z=1$ these lead to peak intensities of $\simeq 40 D^{4} \mu \mathrm{Jy}$ of bulk electrostatic bremsstrahlung near $5 D \mathrm{GHz}, \simeq 10^{5} D^{4} \mathrm{Jy}$ of relativistic bremsstrahlung in the optical band, and $\simeq 100 D^{4} \mathrm{Jy}$ of synchrotron radiation in the infrared band.

\section{Summary and conclusions}

The interaction of collimated relativistic hadronic (Pohl et al. 2002) and leptonic (Schlickeiser et al. 2002) jet outflows from active galactic nuclei with the surrounding interstellar and intergalactic medium generates by two-stream instabilities huge intensities of subluminal electrostatic (Langmuir) wave fields in the co-moving outflow region with energy densities larger 
than the energy density in the magnetic field. We therefore have revisited electrostatic bremsstrahlung, i.e. the inverse Compton scattering of electrostatic waves by energetic positrons and electrons to photons $\left(l+e \rightarrow t+e^{\prime}\right)$, as an alternative nonthermal radiation process to synchrotron radiation which in the literature so far has been the standard low-frequency radiation process for radiation. It is known that this radiation process has similar polarisation properties as synchrotron radiation. By calculating Doppler boosted luminosity spectra in case of leptonic outflows we demonstrate that in jet outflow sources electrostatic bremsstrahlung by swept-up relativistic electrons is stronger than synchrotron radiation from the same electrons in the radio to optical frequency band, whereas the optically thick bulk electrostatic bremsstrahlung from the non-relativistic pair outflow plasma is typically a factor $10^{10}$ smaller.

We have based our analysis on photon angle integrated monochromatic approximations of the electrostatic bremsstrahlung power and synchrotron radiation power in a plasma, which are constructed from moments of the respective radiation powers of single electrons and positrons. The calculated moments hold both for superluminal and subluminal phase velocities of the target electrostatic plasma waves. Specialising to the specific subluminal electrostatic wave distribution and the swept-up relaivistic electrons in the leptonic jet model of Schlickeiser et al. (2002) we calculate the expected Doppler boosted radiation intensities from bulk electrostatic bremsstrahlung, relativistic electron bremsstrahlung and synchrotron radiation. This required to investigate in detail (i) the equilibrium distribution of the radiating electrons and (ii) the emission and absorption coefficients of each radiation process. For standard inner jet outflow parameters we showed that the optical depth is determined solely by bulk electrostatic bremsstrahlung. In consequence, all emitted intensities are optically thick at frequencies below $v_{\mathrm{c}}^{*} \simeq 10 v_{\mathrm{p}}^{*}$. In this frequency range, bulk electrostatic bremsstrahlung dominates. Above $v_{\mathrm{c}}^{*}$, relativistic electrostatic bremsstrahlung and synchrotron radiation are optically thin, extending up to optical and infrared frequencies, respectively, which peak intensities for source at redshift $z=1$ in the range of $10^{5} \mathrm{Jy}$ (electrostatic bremsstrahlung) and $100 \mathrm{Jy}$ (synchrotron radiation) for standard jet outflow parameters.

An attractive feature of the electrostatic bremsstrahlung mechanism is the fact that the interaction of the jet outflow with the surrounding ambient medium generates both the target electrostatic plasma waves and the radiating swept-up relativistic electrons. It does not rely on the existence of large intrinsic ordered magnetic fields to account for strong emission due to the synchrotron radiation process. This might be of importance also (i) for the recently detected strongly polarised $\gamma$ ray emission from the gamma-ray burst of 6 December 2002 (Coburn \& Boggs 2003) and (ii) for the nonthermal emission far away from the central jet engine as e.g. in case of the X-ray detected jet emission in PKS 0637-752 (Schwartz et al. 2000) and 3C 273 (Marshall et al. 2001). In future work we will apply the electrostatic bremsstrahlung mechanism to these and similar Chandra sources. It also will be of interest to take the electrostatic bremsstrahlung radiation fields as target fields for the inverse Compton radiation process
(electrostatic-bremsstrahlung-self-Compton mechanism) in anlogy to the well-explored synchrotron-self-Compton process.

Acknowledgements. I thank Martin Pohl and Urs Schaefer-Rolfs for the careful reading of the manuscript and many helpful comments. I am grateful to the referee for his constructive and helpful comments. This work was partially supported by the Deutsche Forschungsgemeinschaft through Sonderforschungbereich 591.

\section{Appendix A: Calculation of two integrals}

Introducing the functions

$A_{1}(t) \equiv \frac{1}{(1-\beta t)^{3}}-\frac{\left(1-\beta^{2}\right)\left(1-t^{2}\right)}{2(1-\beta t)^{5}}$

and

$A_{2}(t) \equiv \frac{\beta^{2}}{(1-\beta t)^{3}}+\frac{\left(1-\beta^{2}\right)}{(1-\beta t)^{5}}\left[\beta^{2}-\frac{1}{2}-2 \beta t+\frac{3}{2} t^{2}\right]$

and setting $t=\cos \psi, L=\cos \psi_{\mathrm{L}}$, the integrals (35) and (40) read

$I_{1}=\int_{-1}^{1} \mathrm{~d} t A_{1}(t)-L^{2} \int_{-1}^{1} A_{2}(t)$

and

$I_{2}=\int_{-1}^{1} \mathrm{~d} t \frac{A_{1}(t)}{1-\beta t}-L^{2} \int_{-1}^{1} \mathrm{~d} t \frac{A_{2}(t)}{1-\beta t}$

The four $t$-integrals are directly related to the integral

$$
\begin{aligned}
j_{n}(\beta) & \equiv \int_{-1}^{1} \frac{\mathrm{d}(\cos \psi)}{(1-\beta \cos \phi)^{n}} \\
& =\frac{1}{(n-1) \beta\left(1-\beta^{2}\right)^{n-1}}\left[(1+\beta)^{n-1}-(1-\beta)^{n-1}\right]
\end{aligned}
$$

or partial derivatives of $j_{n}(\beta)$ with respect to $\beta$. With $\gamma=(1-$ $\left.\beta^{2}\right)^{-1 / 2}$ we derive

$$
\begin{aligned}
\int_{-1}^{1} \mathrm{~d} t A_{1}(t)= & j_{3}(\beta)+\frac{1-\beta^{2}}{24} \frac{\partial^{2} j_{3}(\beta)}{\partial \beta^{2}}-\frac{1-\beta^{2}}{2} j_{5}(\beta) \\
= & 2 \gamma^{4}+\frac{\left(1+5 \beta^{2}\right) \gamma^{6}}{3}-\gamma^{6}\left(1+\beta^{2}\right)=\frac{4}{3} \gamma^{4}, \quad(134) \\
\int_{-1}^{1} \mathrm{~d} t A_{2}(t)= & \beta^{2} j_{3}(\beta)+\left(1-\beta^{2}\right)\left[\frac{2 \beta^{2}-1}{2} j_{5}(\beta)-\frac{\beta}{2} \frac{\partial j_{4}(\beta)}{\partial \beta}\right. \\
& \left.+\frac{1}{8} \frac{\partial^{2} j_{3}(\beta)}{\partial \beta^{2}}\right]=2 \beta^{2} \gamma^{4}+\frac{\gamma^{6}}{3}\left[3\left(2 \beta^{2}-1\right)\left(1+\beta^{2}\right)\right. \\
& \left.-4 \beta^{2}\left(5+\beta^{2}\right)+3\left(1+5 \beta^{2}\right)\right]=\frac{4}{3} \beta^{2} \gamma^{4}
\end{aligned}
$$

$$
\begin{aligned}
\int_{-1}^{1} \mathrm{~d} t \frac{A_{1}(t)}{1-\beta t}= & j_{4}(\beta)+\frac{1-\beta^{2}}{40} \frac{\partial^{2} j_{4}(\beta)}{\partial \beta^{2}}-\frac{1-\beta^{2}}{2} j_{6}(\beta) \\
= & 2 \gamma^{6}\left(1+\frac{\beta^{2}}{3}\right)+\frac{\left(5+38 \beta^{2}+5 \beta^{4}\right) \gamma^{8}}{15} \\
& -\frac{\left(5+10 \beta^{2}+\beta^{4}\right) \gamma^{8}}{5}=\frac{4\left(5+2 \beta^{2}\right)}{15} \gamma^{6}
\end{aligned}
$$


and

$$
\begin{aligned}
\int_{-1}^{1} \mathrm{~d} t \frac{A_{2}(t)}{1-\beta t}= & \beta^{2} j_{4}(\beta)+\left(1-\beta^{2}\right)\left[\frac{2 \beta^{2}-1}{2} j_{6}(\beta)\right. \\
& \left.-\frac{2 \beta}{5} \frac{\partial j_{5}(\beta)}{\partial \beta}+\frac{3}{40} \frac{\partial^{2} j_{4}(\beta)}{\partial \beta^{2}}\right] \\
= & 2 \beta^{2} \gamma^{6}\left(1+\frac{\beta^{2}}{3}\right) \\
& +\frac{\gamma^{8}}{5}\left[\left(2 \beta^{2}-1\right)\left(\beta^{4}+10 \beta^{2}+5\right)\right. \\
& \left.-8 \beta^{2}\left(5+3 \beta^{2}\right)+5+38 \beta^{2}+5 \beta^{4}\right] \\
= & \frac{4}{15} \beta^{2}\left(6+\beta^{2}\right) \gamma^{6} .
\end{aligned}
$$

\section{Appendix B: Monochromatic approximation of synchrotron radiation in a plasma}

We start from the pitch-angle averaged formula for the synchrotron power of a single electron in a plasma (Crusius \& Schlickeiser 1986, 1988)

$p_{\text {syp }}(v, \gamma)=q_{1} \frac{v}{\gamma^{2}}\left[1+\left(\frac{v_{\mathrm{p}} \gamma}{v}\right)^{2}\right] C S(x)$

with $q_{1}=2 e^{2} \pi / \sqrt{3 c}$,

$x=\frac{2 v}{3 v_{0} \gamma^{2}}\left[1+\left(\frac{v_{\mathrm{p}} \gamma}{v}\right)^{2}\right]^{3 / 2}$

and in terms of Whittaker's function

$C S(x)=W_{0, \frac{4}{3}}(x) W_{0, \frac{1}{3}}(x)-W_{\frac{1}{2}, \frac{5}{6}}(x) W_{-\frac{1}{2}, \frac{5}{6}}(x)$.

It is convenient to introduce the dimensionless frequency

$f=\frac{v}{v_{\mathrm{p}} \gamma}$

and the ratio

$g=\frac{\gamma}{\gamma_{\mathrm{R}}}=\frac{3 v_{0} \gamma}{2 v_{\mathrm{p}}}$

As in the case of electrostatic bremsstrahlung the monochromatic approximation is constructed from the two moments

$M_{0}(\gamma)=\int_{0}^{\infty} \mathrm{d} v p_{\text {syp }}(v, \gamma)=\gamma v_{\mathrm{p}} \int_{0}^{\infty} \mathrm{d} f p_{\text {syp }}(f, \gamma)$

and

$M_{1}(\gamma)=\int_{0}^{\infty} \mathrm{d} v v p_{\text {syp }}(v, \gamma)=\gamma^{2} v_{\mathrm{p}}^{2} \int_{0}^{\infty} \mathrm{d} f f p_{\text {syp }}(f, \gamma)$

where

$p_{\text {syp }}(f, \gamma)=q_{1} \frac{v_{\mathrm{p}}}{\gamma} f\left[1+f^{-2}\right] C S\left(\frac{f}{g}\left(1+f^{-2}\right)^{3 / 2}\right)$.

The zeroth moment (143) gives the total synchrotron power

$P_{\text {syp }}=-m c^{2} \dot{\gamma}_{\text {syp }}=M_{0}(\gamma)$, whereas the ratio of the moments (144) and (143) yields the mean synchrotron frequency as

$<v>(\gamma)=\frac{M_{1}(\gamma)}{M_{0}(\gamma)}=\gamma v_{\mathrm{p}} \frac{H(g)}{E(g)}$

with the two integrals

$H(g) \equiv \int_{0}^{\infty} \mathrm{d} f\left(f^{2}+1\right) C S(x)$

and

$E(g) \equiv \int_{0}^{\infty} \mathrm{d} f\left(f+f^{-1}\right) C S(x)$

where

$x=\frac{f}{g}\left(1+f^{-2}\right)^{3 / 2}$.

The monochromatic approximation then reads

$p_{\text {syp }, \text { mono }}(v, \gamma)=m c^{2}\left|\dot{\gamma}_{\text {syp }}\right| \delta(v-<v>(\gamma))$

The two integrals (148) and (149) are calculated approximately by noting that

$x \simeq \begin{cases}\frac{1}{g f^{2}} & \text { for } f \leq 1 \\ \frac{f}{g} & \text { for } f>1\end{cases}$

so that after obvious changes of the variable of integration

$$
\begin{aligned}
H(g) \simeq & \int_{0}^{1} \mathrm{~d} f\left(f^{2}+1\right) C S\left(\frac{1}{g f^{2}}\right)+\int_{1}^{\infty} \mathrm{d} f\left(f^{2}+1\right) C S\left(\frac{f}{g}\right) \\
= & \frac{1}{2 g^{3 / 2}} \int_{g^{-1}}^{\infty} \mathrm{d} u u^{-5 / 2} C S(u)+\frac{1}{2 g^{1 / 2}} \int_{g^{-1}}^{\infty} \mathrm{d} u u^{-3 / 2} C S(u) \\
& +g \int_{g^{-1}}^{\infty} \mathrm{d} u C S(u)+g^{3} \int_{g^{-1}}^{\infty} \mathrm{d} u u^{2} C S(u)
\end{aligned}
$$

and

$$
\begin{aligned}
E(g) \simeq & \int_{0}^{1} \mathrm{~d} f\left(f+f^{-1}\right) C S\left(\frac{1}{g f^{2}}\right) \\
& +\int_{1}^{\infty} \mathrm{d} f\left(f+f^{-1}\right) C S\left(\frac{f}{g}\right) \\
= & \frac{1}{2 g} \int_{g^{-1}}^{\infty} \mathrm{d} u u^{-2} C S(u) \\
& +\frac{3}{2} \int_{g^{-1}}^{\infty} \mathrm{d} u u^{-1} C S(u)+g^{2} \int_{g^{-1}}^{\infty} \mathrm{d} u u C S(u) .
\end{aligned}
$$

\section{B.1. Plasma case $g \ll 1$}

In the plasma case $g<<1$, corresponding according to Eq. (142) to Lorentz factors $\gamma<<\gamma_{\mathrm{R}}$, the lower integration boundary $g^{-1}>>1$ in the integrals (153) and (154) is much larger than unity, and we may use the asymptotic behaviour (Crusius \& Schlickeiser 1986)

$C S(u>>1) \simeq u^{-1} \exp (-u)$. 
All integrals can then be expressed in terms of confluent hypergeometric functions of the second kind (Abramowitz \& Stegun 1965), yielding

$H(g<<1) \simeq g \exp (-1 / g)\left[\frac{1}{2} U\left(1,-\frac{3}{2}, g^{-1}\right)+\frac{1}{2} U\left(1,-\frac{1}{2}, g^{-1}\right)\right.$

$$
\left.+U\left(1,1, g^{-1}\right)+U\left(1,3, g^{-1}\right)\right]
$$

With the asymptotic behaviour of the confluent hypergeometric function at large arguments we obtain

$H(g<<1) \simeq 3 g^{2} \exp (-1 / g)$.

Likewise, we find

$E(g<<1) \simeq \frac{5}{2} g^{2} \exp (-1 / g)$

$+\frac{g}{2}(1-4 g) \exp (-1 / g) U\left(1,-1, g^{-1}\right) \simeq 3 g^{2} \exp (-1 / g)$.

As a consequence, the mean photon energy (147) becomes

$<v>\left(\gamma \leq \gamma_{\mathrm{R}}\right) \simeq \frac{M_{1}(\gamma)}{M_{0}(\gamma)}=\gamma v_{\mathrm{p}}$

and the total power (146) is

$$
\begin{aligned}
P_{\text {syp }}\left(\gamma \leq \gamma_{\mathrm{R}}\right) & =q_{1} v_{\mathrm{p}}^{2} E(g) \simeq 3 v_{\mathrm{p}}^{2} g^{2} \exp (-1 / g) \\
& =\frac{3 v_{\mathrm{p}}^{2} \gamma^{2}}{\gamma_{\mathrm{R}}} \exp \left(-\frac{\gamma_{\mathrm{R}}}{\gamma}\right)=\frac{27}{4} q_{1} v_{0}^{2} \gamma^{2} \exp \left(-\frac{\gamma_{\mathrm{R}}}{\gamma}\right) .
\end{aligned}
$$

\section{B.2. Vacuum case $g>>1$}

In the vacuum case $g>>1$, corresponding according to Eq. (142) to Lorentz factors $\gamma>>\gamma_{R}$, inspection of the integrals (153) and (154) shows that in each case the last term provides the dominant contribution so that

$$
\begin{aligned}
H(g>>1) & \simeq g^{3} \int_{g^{-1}}^{\infty} \mathrm{d} u u^{2} C S(u) \\
& \simeq g^{3} \int_{0}^{\infty} \mathrm{d} u u^{2} C S(u)-O\left(g^{-7 / 3}\right) \\
& \simeq g^{3} \int_{0}^{\infty} \mathrm{d} u u^{2} C S(u)
\end{aligned}
$$

and

$$
\begin{aligned}
E(g>>1) & \simeq g^{2} \int_{g^{-1}}^{\infty} \mathrm{d} u u C S(u) \\
& \simeq g^{2} \int_{0}^{\infty} \mathrm{d} u u C S(u)-O\left(g^{-4 / 3}\right) \\
& \simeq g^{2} \int_{0}^{\infty} \mathrm{d} u u C S(u) .
\end{aligned}
$$

Both remaining integrals can be solved exactly following the treatment in Eqs. (A11)-(A17) of (Crusius \& Schlickeiser 1988) yielding

$H(g>>1) \simeq \frac{55}{12 \times 36} g^{3} \Gamma\left[\frac{1}{6}\right] \Gamma\left[\frac{5}{6}\right]$, and

$$
E(g>>1) \simeq \frac{16}{27 \pi} g^{2} \Gamma\left[\frac{1}{3}\right] \Gamma\left[\frac{2}{3}\right]
$$

so that the mean photon energy (147) in this case becomes

$<v>\left(\gamma \leq \gamma_{\mathrm{R}}\right) \simeq \frac{M_{1}(\gamma)}{M_{0}(\gamma)}=\frac{55 \sqrt{3} \pi}{256} \gamma v_{\mathrm{p}} g=1.169 v_{\mathrm{p}} \frac{\gamma^{2}}{\gamma_{\mathrm{R}}}$.

For the total power (146) we find

$$
\begin{aligned}
P_{\text {syp }}\left(\gamma>\gamma_{\mathrm{R}}\right) & =q_{1} v_{\mathrm{p}}^{2} E(g) \simeq \frac{32 \pi^{2}}{27 \sqrt{3}} q_{1} v_{\mathrm{p}}^{2} g^{2} \\
& =\frac{8 \pi^{2}}{3 \sqrt{3}} q_{1} v_{0}^{2} \gamma^{2} .
\end{aligned}
$$

\section{B.3. Interpolation formula}

Equations (158) and (164) can be combined to the interpolation formula

$<v>\simeq v_{\mathrm{p}} \gamma\left[1+\frac{\gamma}{\gamma_{\mathrm{R}}}\right]$

which holds for all Lorentz factors. Likewise Eqs. (160) and (165) can be combined to

$P_{\text {syp }}(\gamma) \simeq 3 q_{1} v_{\mathrm{p}}^{2} g^{2} \exp \left(-\frac{1}{g}\right)=\frac{27 q_{1}}{4} v_{0}^{2} \gamma^{2} \exp \left(-\frac{\gamma_{\mathrm{R}}}{\gamma}\right)$.

These two interpolation formulas yield the monochromatic approximation (93) for synchrotron radiation in a plasma.

\section{References}

Abramowitz, M., \& Stegun, I. A. 1965, Handbook of Mathematical Functions (Washington: National Bureau of Standards)

Bekefi, G. 1966, Radiation Processes in Plasmas (New York: John Wiley)

Bloom, S. D., \& Marscher, A. P. 1996, ApJ, 461, 657

Böttcher, M. 2002, in Gamma-Ray Astrophysics through Multiwavelength Experiments, Bull. Astron. Soc. India 30, 115

Böttcher, M., Mause, H., \& Schlickeiser, R. 1997, A\&A, 324, 395

Böttcher, M., Schlickeiser, R., \& Marra, A. 2001, ApJ, 563, 71

Chiuderi, C., \& Veltri, P. 1974, A\&A, 30, 265

Coburn, W., \& Boggs, S. E. 2003, Nature, 423, 415

Colgate, S. A. 1967, ApJ, 150, 163

Crusius, A., \& Schlickeiser, R. 1986, A\&A, 164, L16

Crusius, A., \& Schlickeiser, R. 1988, A\&A, 196, 327

Dermer, C. D., \& Schlickeiser, R. 1993, ApJ, 416, 458

Dermer, C. D., \& Schlickeiser, R. 2002, ApJ, 575, 667

Dermer, C. D., Sturner, S. J., \& Schlickeiser, R. 1997, ApJS, 109, 103

Felten, J. E., \& Morrison, P. 1966, ApJ, 146, 686

Fisk, L. A., Kozlovsky, B., \& Ramaty, R. 1974, ApJ, 180, L35

Gailitis, A. K., \& Tsytovich, V. N. 1964, Soviet Phys. - JETP 19, 1165

Gradshteyn, I. S., \& Ryzhik, I. M. 1965, Tables of Integrals, Series and Products (New York: Academic Press)

Mannheim, K. 1993, A\&A, 269, 67

Maraschi, L., Ghisellini, G., \& Celotti, A. 1992, ApJ, 397, L5

Marshall, H. L., Harris, D. E., Grimes, J. P., et al. 2001, ApJ, 549, L167

Melrose, D. B. 1971, Astrophys. Space Sci., 10, 197 
Melrose, D. B. 1980, Plasma Astrophysics: Nonthermal Processes in Diffuse Magnetized Plasmas, vol. I (New York: Gordon and Breach Science Publ.)

Möbius, E., Hovestadt, D., Klecker, B., et al. 1985, Nature, 318, 426

Pohl, M., Lerche, I., \& Schlickeiser, R. 2002, A\&A, 383, 309

Pohl, M., \& Schlickeiser, R. 2000, A\&A, 354, 395

Rucinski, D., Cummings, A. C., Gloeckler, G., et al. 1996, Space Sci. Rev., 78, 73

Rybicki, G. B., \& Lightman, A. P. 1979, Radiative Processes in Astrophysics (New York: Wiley)

Schlickeiser, R., \& Crusius, A. 1988, ApJ, 328, 578
Schlickeiser, R., Vainio, R., Böttcher, M., et al. 2002, A\&A, 393, 69

Schlickeiser, R. 1996, Space Sci. Rev., 75, 299

Schwartz, D. A., Marshall, H. L., Lovell, J. E., et al. 2000, ApJ, 540, L69

Sikora, M., Begelman, M. C., \& Rees, M. J. 1994, ApJ, 421, 153

Sikora, M., \& Madejski, G. 2002, in Current High Energy Emission around Black Holes, ed. C.-H. Lee, \& H.-Y. Chang (Singapore: World Scientific Publishing), 132

Tavecchio, F., Maraschi, L., \& Ghisellini, G. 1998, ApJ, 509, 608d

Vainio, R., Pohl, M., \& Schlickeiser, R. 2003, A\&A, submitted

Windsor, R. A., \& Kellogg, P. J. 1967, ApJ, 190, 167 